\title{
The influence of landslide shape and continental shelf on landslide generated tsunamis along a plane beach
}

\author{
E. Renzi ${ }^{1, *}$ and P. Sammarco ${ }^{1}$ \\ ${ }^{1}$ Università degli Studi di Roma Tor Vergata, Dipartimento di Ingegneria Civile, Via del Politecnico 1, 00133 Rome, Italy \\ *now at: UCD School of Mathematical Sciences, University College Dublin, Belfield, Dublin 4, Ireland
}

Correspondence to: E. Renzi (emiliano.renzi@ucd.ie)

Received: 13 October 2011 - Revised: 8 February 2012 - Accepted: 11 March 2012 - Published: 16 May 2012

\begin{abstract}
This work proposes an advancement in analytical modelling of landslide tsunamis propagating along a plane beach. It is divided into two parts. In the first one, the analytical two-horizontal-dimension model of Sammarco and Renzi (2008) for tsunamis generated by a Gaussian-shaped landslide on a plane beach is revised and extended to realistic landslide shapes. The influence of finiteness and shape of the slide on the propagating waves is investigated and discussed. In the second part, a new model of landslide tsunamis propagating along a semi-plane beach is devised to analyse the role of the continental platform in attenuating the wave amplitude along the shoreline. With these parameters taken into account, the fit with available experimental data is enhanced and the model completed.
\end{abstract}

\section{Introduction}

The recent Sendai tsunami in Japan has shown how destructive such an event is for coastal communities (see Li et al., 2011). Catastrophic tsunamis can be generated by a number of natural events like earthquakes and submerged or subaerial landslides. While excellent advancements have been made in understanding earthquake tsunamis, knowledge of the generation and propagation of landslide tsunamis is instead still fragmentary (see Liu et al., 2005). The most challenging issue is that landslide tsunamis are not generated instantaneously as earthquake tsunamis, but strongly depend on the time history of the seafloor deformation. As a consequence, these events cannot be investigated by transferring to the free-surface a "hot start" initial condition due to the ground movement (see Sammarco and Renzi, 2008). Indeed, at the state of the art, the main gap in modelling landslide tsunamis seems to be the scarcity of analytical models that take into account the prolonged interaction between land- slide and water (see Lynett and Liu, 2005). The model of Sammarco and Renzi (2008) on landslide tsunamis propagating along a plane beach (SR model in the following) contributed to fill this gap with a specific insight on the coupled dynamics of landslide motion and wave field generation. By solving the 2-D horizontal wave field, Sammarco and Renzi (2008) investigated the general behaviour of the system and showed that after a short time following the landslide generation, the wave motion is made by transient edge waves travelling along the shoreline, the offshore motion being practically absent. The wave field shows a strong dispersive behaviour, with longer waves travelling faster and the highest crests shifted towards the middle of the wave train. Despite being one of the few three-dimensional models available in the literature, the SR model might be further improved by removing some of its limiting assumptions. First, the authors modelled the landslide as a double Gaussian-shaped, rigid body, starting its motion from a fixed position (corresponding to a half-submerged slide) and moving along the incline with given velocity (about $1 \mathrm{~m} \mathrm{~s}^{-1}$ ). Therefore, the SR model, yet providing a good description of the tsunami generation and propagation mechanisms, does not describe the influence of the slide initial position and velocity on the generated wave field. Furthermore, the double Gaussian slide, with its infinite length, is not completely representative of a real landslide shape of finite length. Second, the indefinite plane beach of Sammarco and Renzi (2008) extends to infinite depth, thus, being not representative of realistic bathymetries, where the sloping beach eventually connects to a flat continental platform. To overcome these drawbacks, in the present work we extend the SR model to investigate the influence of the landslide shape and physical parameters and of the continental platform on tsunamis propagating along a plane beach. Contemporaneously, a statistical analysis based on the extended SR model is being carried out by 


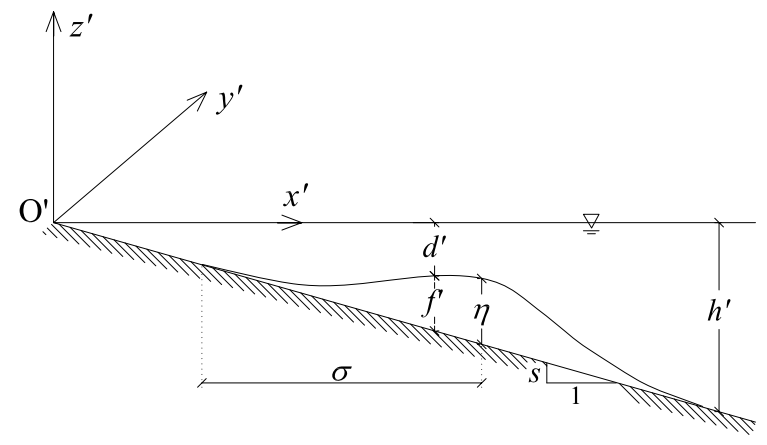

Fig. 1. The fluid domain in physical coordinates; $\eta$ and $\sigma$ are respectively the slide maximum vertical thickness and characteristic horizontal length, depending on the shape of the landslide.

Sarri et al. (2012).

In Sect. 2 the analytical two-horizontal dimension (2HD) model is deduced for a general shape and law of motion of the slide. Then the model is applied to investigate the parametric dependence of the generated wave field on the slide starting position and moving speed along the incline. Also, the influence of the shape is investigated by considering a double parabolic landslide of finite length with arbitrary speed and initial position. Comparison is made between the two models to show how the slide finiteness influences the generated wave field. In Sect. 3 an analytical 2HD model is developed to investigate the influence of the continental platform on landslide tsunamis propagating along a semi-plane beach. In both sections results are discussed and the freesurface elevation time series are calculated, showing excellent agreement with available experimental data.

\section{Influence of the landslide shape and physical parameters}

\subsection{Position of the problem}

Referring to Fig. 1, let us consider a plane beach with constant slope $s$ and define a Cartesian reference system of coordinates $\left(\mathrm{O}^{\prime}, x^{\prime}, y^{\prime}, z^{\prime}\right)$, with the $y^{\prime}$-axis along the mean shoreline, the $z^{\prime}$ axis pointing vertically upwards and water in the region $x^{\prime}>0$. We assume that the landslide originates in a neighbourhood of the origin $\mathrm{O}^{\prime}$ and that it is symmetric with respect to the $y^{\prime}$-axis; the induced wave field is also symmetric in $y^{\prime}$, hence, we shall solve the equation of motion in $y^{\prime}>0$ only. Now, let $\eta$ and $\sigma$ be, respectively, the maximum vertical height and the characteristic horizontal length of the landslide. Let us further assume that the slope is mild, i.e. $s \ll 1$, and that the slide is thin, with $\eta / \sigma \ll 1$. Under these assumptions, we can employ the linear long-wave equation for forced waves on a uniformly sloping beach to describe the physics of the problem (Liu et al., 2003):

$\frac{\partial^{2} \zeta^{\prime}}{\partial t^{\prime 2}}-g \nabla \cdot\left(h^{\prime} \nabla \zeta^{\prime}\right)=\frac{\partial^{2} f^{\prime}}{\partial t^{\prime 2}}$.

In the latter, $\nabla(\cdot)=\left[\partial(\cdot) / \partial x^{\prime}, \partial(\cdot) / \partial y^{\prime}\right]$ is the nabla operator, and $\zeta^{\prime}\left(x^{\prime}, y^{\prime}, t^{\prime}\right)$ the free-surface elevation; $g$ is the acceleration due to gravity; $t^{\prime}$ denotes time and $d^{\prime}=h^{\prime}-f^{\prime}\left(x^{\prime}, y^{\prime}, t^{\prime}\right)$ the bottom depth, measured with respect to the mean water level $z^{\prime}=0$. In the previous expression $h^{\prime}=s x^{\prime}$ is the undisturbed bottom depth, while $f^{\prime}\left(x^{\prime}, y^{\prime}, t^{\prime}\right)$ is a time-dependent perturbation of the seafloor, which represents the landslide moving on the beach (see Fig. 1). Upon introduction of the following non-dimensional variables

$$
(x, y)=\left(x^{\prime}, y^{\prime}\right) / \sigma, \quad t=\sqrt{g s / \sigma} t^{\prime}, \quad(\zeta, f)=\left(\zeta^{\prime}, f^{\prime}\right) / \eta,
$$

Equation (1) becomes

$x \zeta_{x x}+\zeta_{x}+x \zeta_{y y}=\zeta_{t t}-f_{t t}$,

where the subscripts denote differentiation with respect to the relevant variable. The free-surface elevation $\zeta(x, y, t)$ must be bounded at the shoreline $x=0$ and as $x \rightarrow \infty$. Finally, we require null initial free-surface elevation and velocity, i.e. $\zeta(x, y, 0)=0$ and $\zeta_{t}(x, y, 0)=0$. The complete analytical solution of this boundary-value problem for the free-surface elevation $\zeta(x, y, t)$ has been obtained by Sammarco and Renzi (2008) for a generic bottom perturbation $f(x, y, t)$. Here, we shall retrace the core passages of their analysis. Application of the cosine Fourier transform pair along $y$

$\hat{\zeta}(x, k, t)=\int_{0}^{\infty} \zeta(x, y, t) \cos k y d y, \zeta=\frac{2}{\pi} \int_{0}^{\infty} \hat{\zeta} \cos k y d k(4)$

and the method of variation of parameters to the forced Eq. (3) yield

$\zeta(x, y, t)=\frac{2}{\pi} \sum_{n=0}^{\infty} \int_{0}^{\infty} e^{-k x} L_{n}(2 k x) T_{n}(k, t) \cos k y d k$

for the free-surface elevation. In the latter expression, $L_{n}$ are the Laguerre polynomials of zero-th order and degree $n \in \mathbb{N}$, corresponding to the free spatial oscillations (eigensolutions) of the plane beach (see Mei et al., 2005). The $T_{n}$ s in Eq. (5) are given by

$T_{n}(k, t)=\frac{2 k}{\omega_{n}} \int_{0}^{\infty} e^{-k \alpha} L_{n}(2 k \alpha) I_{n}(\alpha, k, t) d \alpha$,

with

$I_{n}(\alpha, k, t)=\int_{0}^{t} \hat{f}_{\tau \tau}(\alpha, k, \tau) \sin \left[\omega_{n}(t-\tau)\right] d \tau$,

where

$\omega_{n}=\sqrt{k(2 n+1)}$ 

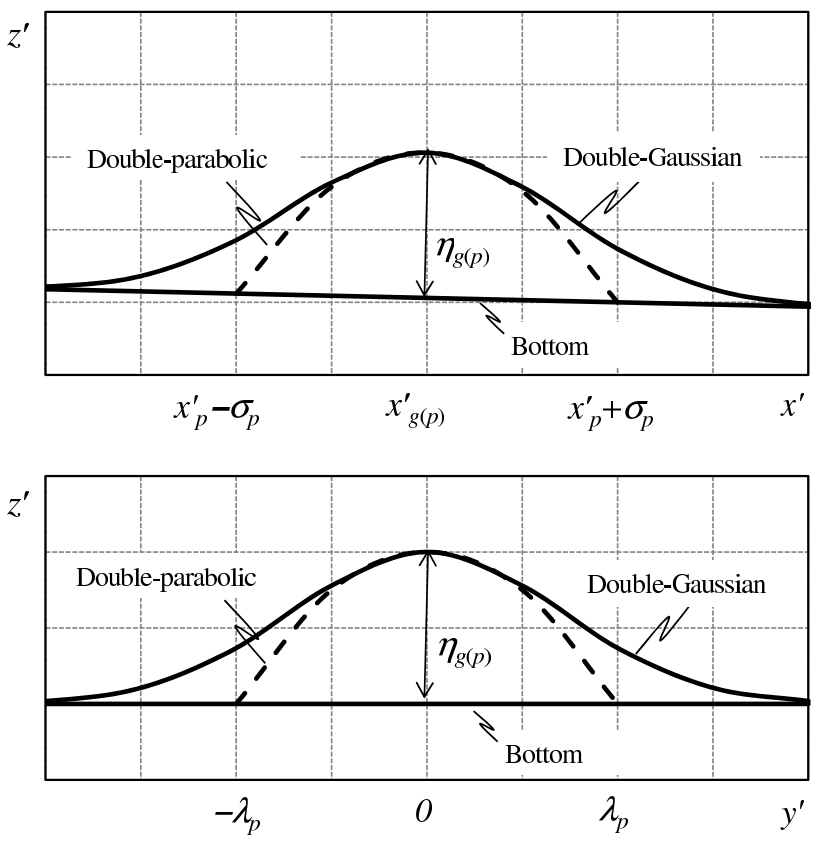

Fig. 2. Vertical cross sections of the double Gaussian landslide (solid line) and the double parabolic slide (dashed lines) in physical variables in the $\left(x^{\prime}, z^{\prime}\right)$ plane (upper panel) and in the $\left(y^{\prime}, z^{\prime}\right)$ plane (lower panel). Here $\sigma_{g}=\sigma_{p}, \eta_{g}=\eta_{p}$ and vertical dimensions are exaggerated for easiness of reading.

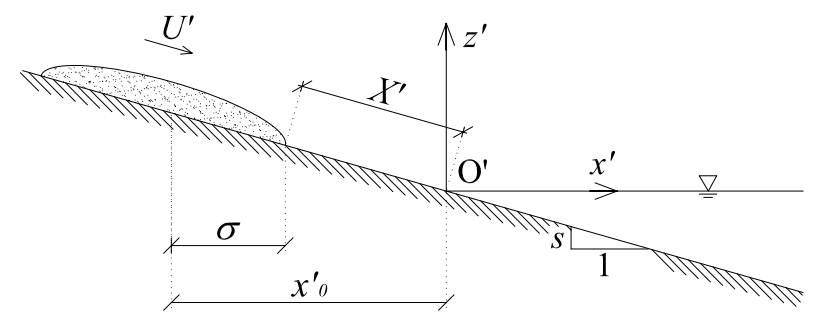

Fig. 3. Ellipsoidal slide used in the experiments of Di Risio et al. (2009). The initial position of the centroid is $x_{0}^{\prime} ; X^{\prime}$ represents the landslide release distance, while $U^{\prime}$ is the slide velocity along the incline.

are the motion eigenfrequencies in the transformed space. Each of the $\omega_{n}$ is associated with the $n$-th modal Laguerre eigenfunction $L_{n}$. Finally in Eq. (7) $\hat{f}_{\tau \tau}$ is the second-order time derivative of the Fourier transform of the bottom perturbation $f(x, y, \tau)$. Clearly, the $I_{n}$ in Eq. (7) and henceforth the free-surface elevation $\zeta$ in Eq. (5) can be evaluated only after having determined the shape of the slide and its law of motion by imposing an analytical form to the forcing term $f$.

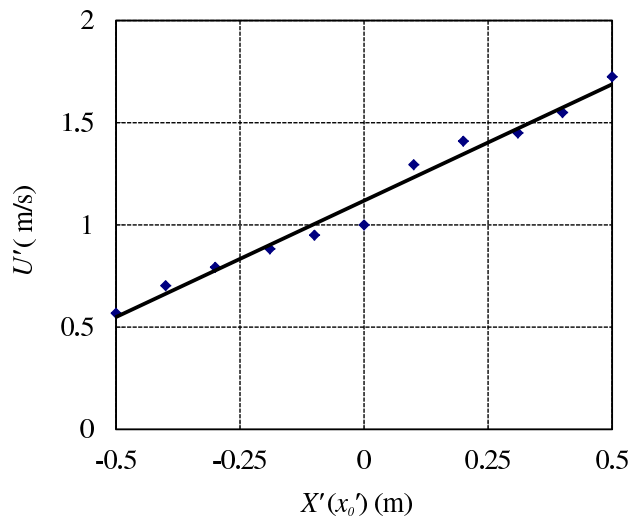

Fig. 4. Experimental relationship between the mean underwater velocity $U^{\prime}$ and the release distance $X^{\prime}$ obtained by Di Risio et al. (2009) for an ellipsoidal landslide. Diamonds show experimental measurements, the bold line the relevant linear regression Eq. (37). Note that $U^{\prime} \approx 1 \mathrm{~m} \mathrm{~s}^{-1}$ for $X^{\prime}=0$.

\subsection{Landslide shape}

Sammarco and Renzi (2008) solved the forced plane-beach problem of Eq. (3) by considering a translating Gaussian seafloor movement, whose kinematic description was given by

$f(x, y, t)=\exp \left[-(x-t)^{2}\right] \exp \left[-(\sigma / \lambda y)^{2}\right]$,

being $\lambda$ the characteristic width of the slide at the shoreline. Expression Eq. (9) represents a double Gaussian-shaped slide moving in the offshore direction at uniform speed $u=1$, whose centroid occupies the position $x=0$ for $t=0$, i.e. at rest. The results provided by the authors are in satisfactory agreement with available experimental data (see Di Risio et al., 2009) for a similar condition of the SR model. However, at a deeper insight, both numerical and experimental results (e.g. Liu et al., 2005; Lynett and Liu, 2005; Di Risio et al., 2009) have shown that the generation and propagation of landslide tsunamis along a sloping beach are sensibly influenced by the shape, the initial position and the speed of the slide. Hence, the expression of the forcing term $f(x, y, t)$ in Eq. (9), yet describing satisfactorily the general behaviour of the system, needs some improvements to be applied to more advanced tsunami forecasting models. In order to investigate the physics not reproduced by the Gaussian slide of the SR model, in this section we shall extend our analysis to two different and more complete landslide shape functions.

First, we retain the double Gaussian shape, but allow for representation of the landslide initial position and mean speed by defining the forcing term as

$f_{g}(x, y, t)=\exp \left[-\left(x-x_{g}-u_{g} t\right)^{2}\right] s_{g}(y)$,

where

$s_{g}(y)=\exp \left[-\left(c_{g} y\right)^{2}\right]$ 
is the lateral spreading function and $c_{g}=\sigma_{g} / \lambda_{g}$ is the lateral spreading factor, the subscript $g$ denoting quantities relevant to the double Gaussian slide. Equations (10) and (11) represent a double Gaussian-shaped slide moving as a rigid body in the offshore direction, with its centroid initially at $x=x_{g}$, and with uniform speed $u=u_{g}$ along $x$. At any time $t$ the centroid is at $(x, y)=\left(x_{g}+u_{g} t, 0\right)$, where the slide thickness is maximum, i.e. $f_{g}=1$ in nondimensional variables. In the following, a landslide for which $x_{g}<0\left(x_{g}>0\right)$ will be referred to as subaerial (submerged), according to the initial position of its centroid. Second, we investigate the influence of the landslide shape and finiteness on the generated wave field by considering a finite-length double parabolic slide, whose shape and motion are described by

$$
\begin{aligned}
f_{p}(x, y, t) & =\left(x-x_{p}-u_{p} t+1\right)\left(x_{p}+u_{p} t+1-x\right) s_{p}(y) \\
& \times H\left(x-x_{p}-u_{p} t+1\right) H\left(x_{p}+u_{p} t+1-x\right) \\
& \times H\left(1 / c_{p}-y\right) .
\end{aligned}
$$

In the latter,

$$
s_{p}(y)=\left(1-c_{p} y\right)\left(1+c_{p} y\right)
$$

is the lateral spreading function and $c_{p}=\sigma_{p} / \lambda_{p}$ the lateral spreading factor, the subscript $p$ denoting quantities relevant to the double parabolic slide. In Eq. (12) the Heaviside step function $H$ is introduced to cut the slide into a finite length along $x$ and $y$; only the half-space $y>0$ is considered due to the symmetry of the problem about $y=0$. Equations (12) and (13) represent a landslide with a finite rectangular footprint and parabolic vertical cross sections about the $x$ and $y$ axes; again $x_{p}$ is the centroid initial position and $u_{p}$ the mean downfall speed of the slide along $x$. In the following, the solution of the forced equation of motion Eq. (3) will be found in terms of the free-surface elevation Eq. (5), and the relevant wave field discussed, for each of the two proposed forcing functions. The vertical cross sections of both the slides are represented in Fig. 2 for easiness of comparison. For the sake of clarity, all the quantities defined above will be referred to with a $g$ subscript for the Gaussian slide and a $p$ subscript for the double parabolic slide.

\subsection{Solution}

With the landslide forcing functions defined by Eqs. (10) and (12) for the double Gaussian and the double parabolic landslide respectively, the integral function $I_{n}$ Eq. (7) and then the free-surface elevation $\zeta$ Eq. (5) can now be determined for each of the two slides. For the Gaussian-shaped landslide, substitution of Eq. (10) into Eq. (7) and integration by parts yield

$$
\begin{array}{r}
I_{g, n}=\omega_{n} \hat{s}_{g}(k)\left\{\left[\omega_{n} a_{g, n}-e^{-\left(\alpha-x_{g}\right)^{2}}\right] \cos \omega_{n} t\right. \\
-\left[\frac{2 u_{g}\left(\alpha-x_{g}\right)}{\omega_{n}} e^{-\left(\alpha-x_{g}\right)^{2}}+\omega_{n} b_{g, n}\right] \sin \omega_{n} t \\
\left.+e^{-\left(\alpha-x_{g}-u_{g} t\right)^{2}}\right\},
\end{array}
$$

where $\hat{s}_{g}(k)=\sqrt{\pi} /\left(2 c_{g}\right) e^{-k^{2} /\left(4 c_{g}^{2}\right)}$ is the cosine Fourier transform of the spreading function $s_{g}(y)$ Eq. (11) and

$$
\begin{array}{r}
a_{g, n}=a_{g, n}(\alpha, k, t)=\frac{\sqrt{\pi}}{2 u_{g}} e^{-\omega_{n}^{2} / 4 u_{g}^{2}} \\
\times \Im\left\{e ^ { \mathrm { i } \omega _ { n } ( \alpha - x _ { g } ) / u _ { g } } \left[\operatorname{erf}\left(\alpha-x_{g}+\mathrm{i} \frac{\omega_{n}}{2 u_{g}}\right)\right.\right. \\
\left.\left.-\operatorname{erf}\left(\alpha-x_{g}-u_{g} t+\mathrm{i} \frac{\omega_{n}}{2 u_{g}}\right)\right]\right\},
\end{array}
$$

while

$$
\begin{array}{r}
b_{g, n}=b_{g, n}(\alpha, k, t)=\frac{\sqrt{\pi}}{2 u_{g}} e^{-\omega_{n}^{2} / 4 u_{g}^{2}} \\
\times \Re\left\{e ^ { \mathrm { i } \omega _ { n } ( \alpha - x _ { g } ) / u _ { g } } \left[\operatorname{erf}\left(\alpha-x_{g}+\mathrm{i} \frac{\omega_{n}}{2 u_{g}}\right)\right.\right. \\
\left.\left.-\operatorname{erf}\left(\alpha-x_{g}-u_{g} t+\mathrm{i} \frac{\omega_{n}}{2 u_{g}}\right)\right]\right\} .
\end{array}
$$

In Eqs. (15) and (16) i is the imaginary unit, while $\Re\{\}$ and $\Im\{\}$ indicate, respectively, the real and imaginary part of \{\} . Substituting Eq. (14) into Eq. (6) for $T_{g, n}$ and then the latter into Eq. (5), we finally obtain the analytical form of the freesurface elevation for the Gaussian landslide:

$$
\begin{array}{r}
\zeta_{g}(x, y, t)=\frac{2}{\pi} \sum_{n=0}^{\infty} \int_{0}^{\infty} e^{-k x} L_{n}(2 k x) \frac{2 k}{\omega_{n}} \times \\
\times \int_{0}^{\infty} e^{-k \alpha} L_{n}(2 k \alpha) \omega_{n} \hat{s}_{g}(k) \\
\times\left\{\left[\omega_{n} a_{g, n}-e^{-\left(\alpha-x_{g}\right)^{2}}\right] \cos \omega_{n} t+\right. \\
-\left[\frac{2 u_{g}\left(\alpha-x_{g}\right)}{\omega_{n}} e^{-\left(\alpha-x_{g}\right)^{2}}+\omega_{n} b_{g, n}\right] \sin \omega_{n} t \\
\left.+e^{-\left(\alpha-x_{g}-u_{g} t\right)^{2}}\right\} d \alpha \cos k y d k .
\end{array}
$$

To get a physical interpretation of Eq. (17), let us now define the integral transform $\mathcal{L}^{n}$ of a given function $u(\alpha, k, t)$ as

$\mathcal{L}^{n}[u](k, t)=\frac{2 k}{\omega_{n}} \int_{0}^{\infty} e^{-k \alpha} L_{n}(2 k \alpha) u(\alpha, k, t) d \alpha$,

so that Eq. (6) can be easily rewritten as $T_{n}=\mathcal{L}^{n}\left[I_{n}\right]$. Hence, by applying the transform $\mathcal{L}^{n}$ to Eq. (14), we can formally rewrite Eq. (17) as $\zeta_{g}=\zeta_{g}^{o}+\zeta_{g}^{e}$, where

$$
\begin{array}{r}
\zeta_{g}^{o}=\frac{2}{\pi} \sum_{n=0}^{\infty} \int_{0}^{\infty} e^{-k x} L_{n}(2 k x)\left[A_{g, n} \cos \omega_{n} t\right. \\
\left.+B_{g, n} \sin \omega_{n} t\right] \cos k y d k,
\end{array}
$$

with

$$
\begin{aligned}
A_{g, n}=A_{g, n}(k, t) & =\omega_{n} \hat{s}_{g} \mathcal{L}^{n}\left[\omega_{n} a_{g, n}(\alpha, k, t)\right. \\
& \left.-e^{-\left(\alpha-x_{g}\right)^{2}}\right]
\end{aligned}
$$



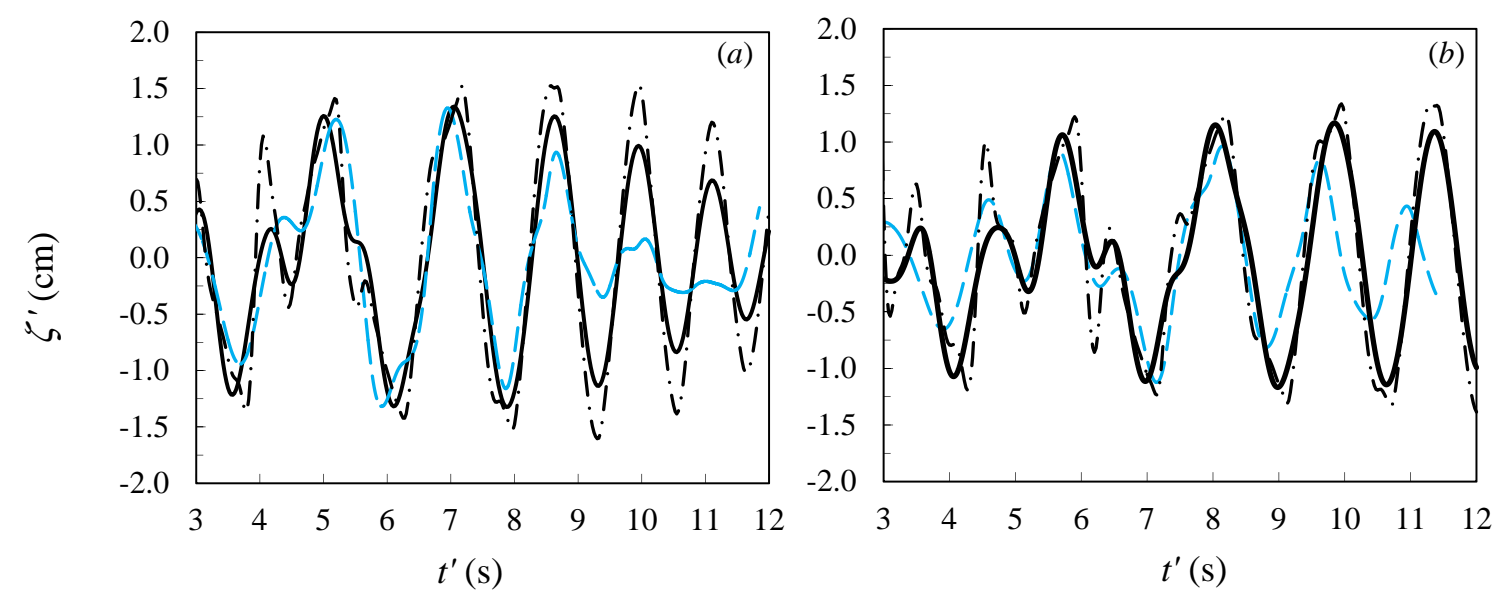

Fig. 5. Free-surface time series at (a) $\left(x^{\prime}, y^{\prime}\right)=(0,3.10 \mathrm{~m})$ and (b) $\left(x^{\prime}, y^{\prime}\right)=(0,4.07 \mathrm{~m})$. The blue dashed line shows the experimental data. The solid line (-) represents the theoretical values for the double Gaussian slide, while the dash-dotted line (-.) refers to the double parabolic slide. For both scenarios, the free-surface elevation is evaluated with the stationary-phase approximation formula and then transformed into dimensional form.

$$
\begin{array}{r}
B_{g, n}=B_{g, n}(k, t)=-\hat{s}_{g} \mathcal{L}^{n}\left[2 u_{g}\left(\alpha-x_{g}\right) e^{-\left(\alpha-x_{g}\right)^{2}}\right. \\
\left.+\omega_{n}^{2} b_{g, n}(\alpha, k, t)\right],
\end{array}
$$

and

$$
\begin{aligned}
\zeta_{g}^{e}= & \frac{2}{\pi} \sum_{n=0}^{\infty} \int_{0}^{\infty} e^{-k x} L_{n}(2 k x) \omega_{n} \hat{s}_{g}(k) \\
& \times \mathcal{L}^{n}\left[e^{-\left(\alpha-x_{g}-u_{g} t\right)^{2}}\right] \cos k y d k
\end{aligned}
$$

The component $\zeta_{g}^{o}$ Eq. (19) describes an oscillatory motion in time, depending on $\cos \omega_{n} t$ and $\sin \omega_{n} t$. Note that at large times $t \gg 1$, the second erf in Eqs. (15) and (16) approaches unity, and the $a_{g, n}$ and $b_{g, n}$ do not depend on time anymore. As a consequence, $A_{g, n}$ Eq. (20) and $B_{g, n}$ Eq. (21) approach limiting values that do not vary with time. The component $\zeta_{g}^{e}$ Eq. (22) fastly decays with time, depending on $\exp \left[-\left(u_{g} t\right)^{2}\right]$. Hence, the landslide generates a wave field made up by an oscillatory and an evanescent component, the latter rapidly vanishing with time. Note that this result is similar in form to the one already obtained by Sammarco and Renzi (2008) for the unit speed Gaussian slide of Eq. (9). In fact, by letting $x_{g}=0$ and $u_{g}=1$, the previous results of the authors are fully recovered. Similarly, by applying the same passages as above to Eq. (12) and after some lengthy algebra, the free-surface elevation relevant to the wave field generated by the double parabolic landslide can be expressed as well as $\zeta_{p}=\zeta_{p}^{o}+\zeta_{p}^{e}$. In the latter expression, the first component is given again by

$$
\begin{array}{r}
\zeta_{p}^{o}=\frac{2}{\pi} \sum_{n=0}^{+\infty} \int_{0}^{\infty} e^{-k x} L_{n}(2 k x)\left[A_{p, n}(k, t) \cos \left(\omega_{n} t\right)\right. \\
\left.+B_{p, n}(k, t) \sin \left(\omega_{n} t\right)\right] \cos (k y) d k
\end{array}
$$

with

$$
\begin{array}{r}
A_{p, n}(k, t)=\omega_{n} \bar{s}_{p}(k) \mathcal{L}^{n}\left[\omega_{n} a_{p, n}-\left(\alpha-x_{p}+1\right)\right. \\
\left.\times\left(x_{p}+1-\alpha\right) H\left(\alpha-x_{p}+1\right) H\left(x_{p}+1-\alpha\right)\right], \\
B_{p, n}(k, t)=-\bar{s}_{p}(k) \mathcal{L}^{n}\left[\omega_{n}^{2} b_{p, n}-2 u_{p}\left(\alpha-x_{p}\right)\right. \\
\left.\quad \times H\left(\alpha-x_{p}+1\right) H\left(x_{p}+1-\alpha\right)\right],
\end{array}
$$

which are the counterparts of the $A_{g, n}$ and $B_{g, n}$ for the Gaussian slide in Eqs. (20) and (21), respectively. Furthermore, in Eqs. (24)-(25) the term

$$
\begin{aligned}
\bar{s}_{p}(k) & =\int_{0}^{\infty} s_{p}(y) H\left(\frac{1}{c_{p}}-y\right) \cos (k y) d y \\
& =\frac{2 c_{p}}{k^{3}}\left[c_{p} \sin \left(\frac{k}{c_{p}}\right)-k \cos \left(\frac{k}{c_{p}}\right)\right]
\end{aligned}
$$

comes from the cosine Fourier transform of the forcing term $f_{p}$ of Eq. (12), while the $a_{p, n}$ and $b_{p, n}$ are given respectively by

$a_{p, n}=\mathfrak{J}\{g(\alpha)\}, \quad b_{p, n}=\Re\{g(\alpha)\}$,

where

$$
\begin{array}{r}
g(\alpha)=\int_{0}^{\infty}\left(\alpha-x_{p}-u_{p} \tau+1\right)\left(x_{p}+u_{p} \tau+1-\alpha\right) \\
\times H\left(\alpha-x_{p}-u_{p} \tau+1\right) H\left(x_{p}+u_{p} \tau+1-\alpha\right) e^{\mathrm{i} \omega_{n} \tau} d \tau .
\end{array}
$$

Within this framework, the $a_{p, n}$ and $b_{p, n}$ in Eq. (26) play the same role as the $a_{g, n}$ and $b_{g, n}$ for the Gaussian slide in Eqs. (15) and (16), respectively. Note also that $\zeta_{p}^{o}$ Eq. (23) 


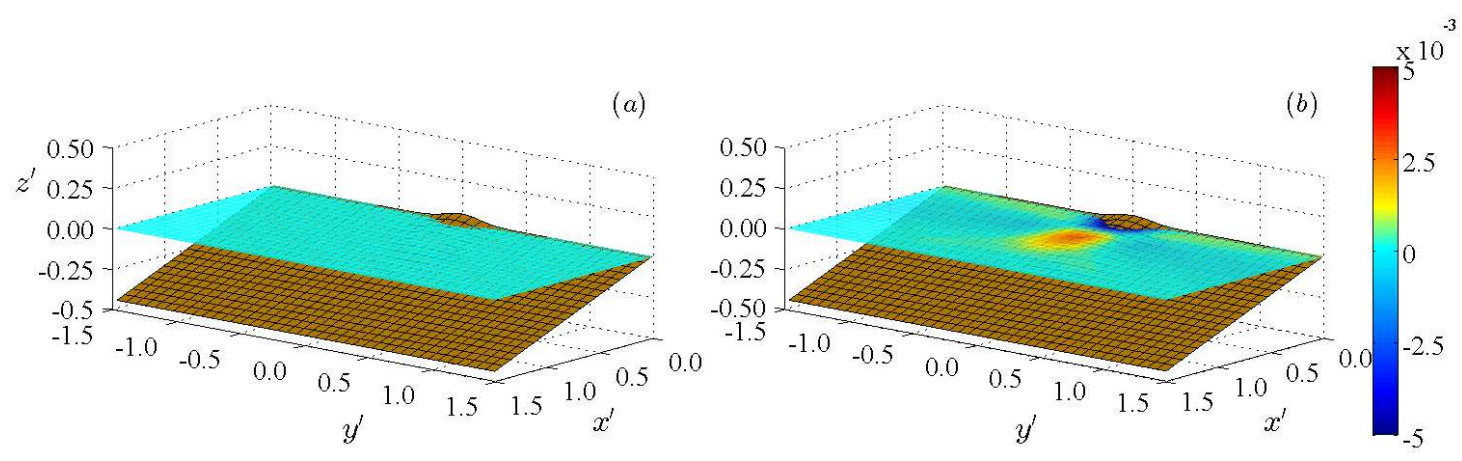

(c)
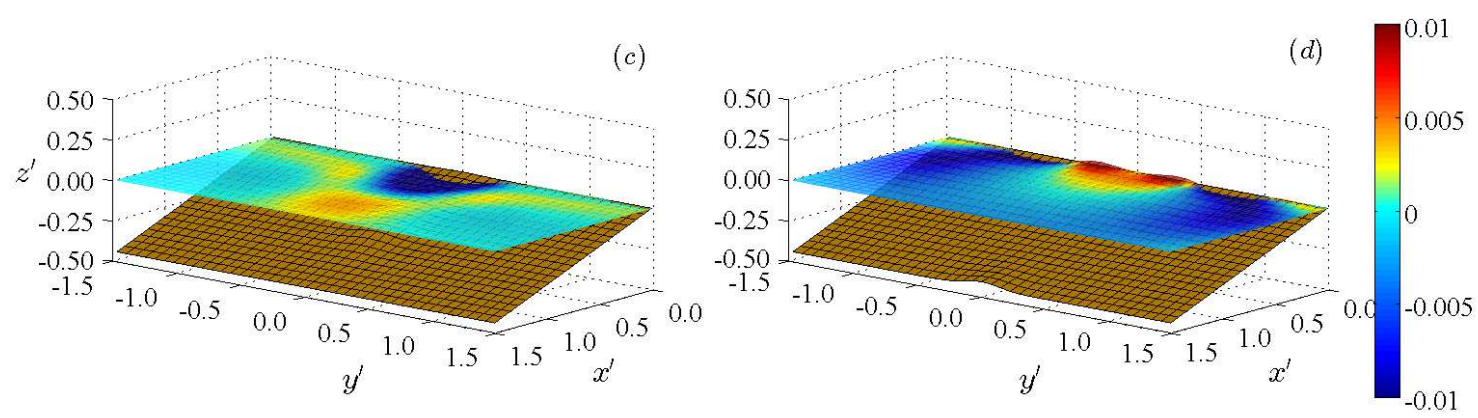

Fig. 6. Snapshots of the free-surface profile in physical variables at times: (a) $t^{\prime}=0 \mathrm{~s}$, (b) $t^{\prime}=0.2 \mathrm{~s}$ (corresponding to $t=0.5$ ), (c) $t^{\prime}=0.5 \mathrm{~s}$ $(t=1.5)$ and (d) $t^{\prime}=1.5 \mathrm{~s}(t=4.5)$. The double Gaussian landslide of parameters $c_{g}=2, x_{g}^{\prime}=0 \mathrm{~m}, u_{g}^{\prime}=1 \mathrm{~m} \mathrm{~s}^{-1}$ is taken as reference model. The first six modes have been considered. All values are in metres.

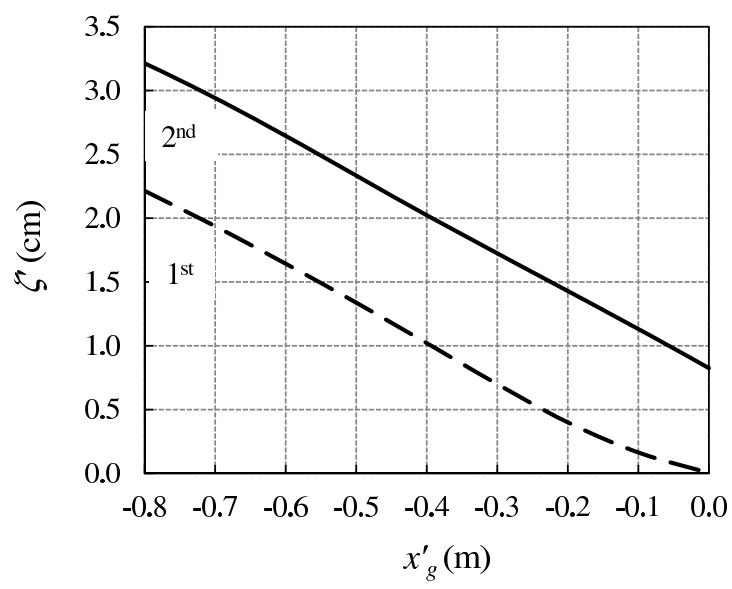

Fig. 7. Influence of the centroid initial position on the wave field. Figure shows the maximum free-surface elevation of the first wave (dashed line - -) and of the second wave (solid line -) vs. $x_{g}^{\prime}$ at a point on the shoreline $\left(y^{\prime}=3.10 \mathrm{~m}\right)$. Equation (39) is used to relate the slide velocity to the centroid initial position. The double Gaussian reference slide of parameters $\sigma_{g}=0.37 \mathrm{~m}, \eta_{g}=0.045 \mathrm{~m}$ and $c_{g}=2$ (see Sect. 2.4) has been considered. has the same formal expression as $\zeta_{g}^{o}$ Eq. (19), i.e. it describes an oscillatory component. Finally, the second term of the decomposition $\zeta_{p}=\zeta_{p}^{o}+\zeta_{p}^{e}$ is obtained as

$$
\begin{array}{r}
\zeta_{p}^{e}=\frac{2}{\pi} \sum_{n=0}^{+\infty} \int_{0}^{\infty} e^{-k x} L_{n}(2 k x) \cos (k y) \omega_{n} \bar{s}_{p}(k) \\
\times \mathcal{L}^{n}\left[\left(\alpha-x_{p}-u_{p} t+1\right)\left(x_{p}+u_{p} t+1-\alpha\right)\right. \\
\left.\times H\left(\alpha-x_{p}-u_{p} t+1\right) \cdot H\left(x_{p}+u_{p} t+1-\alpha\right)\right] d k .
\end{array}
$$

Clearly, in the latter equation at large $t$, the two Heaviside step functions will constrain the domain of integration of $\mathcal{L}^{n}$ between $\alpha_{1}=x_{p}+u_{p} t-1$ and $\alpha_{2}=x_{p}+u_{p} t+1$. Approaching large times $\alpha_{2} / \alpha_{1} \simeq 1$, the domain of integration of $\mathcal{L}^{n}$ in Eq. (27) collapses into a single point and the integral rapidly vanishes: $\zeta_{p}^{e}$ still represents an evanescent term, being the counterpart of $\zeta_{g}^{e}$ Eq. (22) for the Gaussian slide. Hence, the decomposition of the generated wave field into an oscillatory and an evanescent component is a general result, independent of the shape and finiteness of the slide. Computational aspects concerning the numerical evaluation of $\zeta_{g(p)}^{o}$ and $\zeta_{g(p)}^{e}$ are discussed in Appendix B. In the following we shall provide an estimate of the decay of the evanescent terms for both the landslides. 

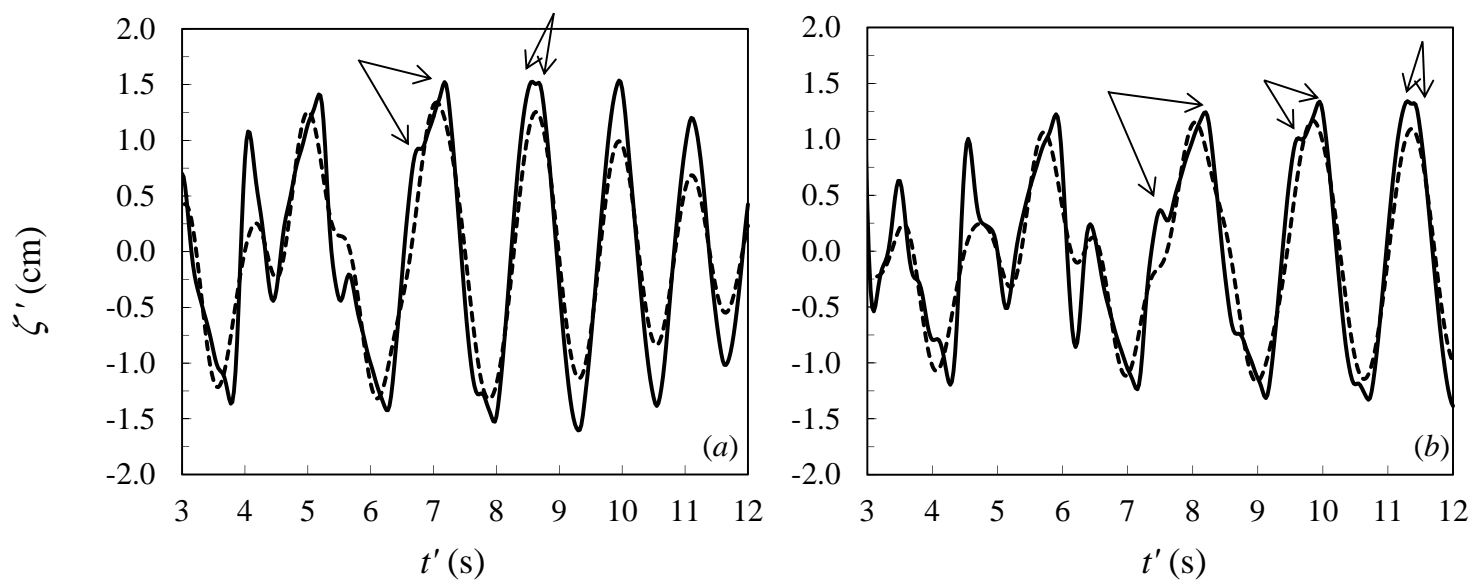

Fig. 8. Free-surface time series in physical variables at $(\mathbf{a})\left(x^{\prime}, y^{\prime}\right)=(0,3.10 \mathrm{~m})$ and $(\mathbf{b})\left(x^{\prime}, y^{\prime}\right)=(0,4.07 \mathrm{~m})$. The bold dashed line represents the values of $\zeta_{g}^{\prime}$ for the infinite double Gaussian landslide, while the continuous line shows the values of $\zeta_{p}^{\prime}$ for the finite-length double parabolic landslide, both evaluated with the stationary-phase approximation formula. The first five modes have been considered for the double Gaussian slide, and the first seven modes for the double parabolic slide. The arrows show the double-crested waves in the perturbation generated by the double parabolic slide.

\subsubsection{Decay of the evanescent component}

An estimate of the decay of the evanescent components can be obtained with an asymptotic analysis at large time. Starting from the Gaussian slide of Eq. (10), consider the $n$ th modal component $\zeta_{g, n}^{e}$ of the evanescent term Eq. (22). When $t$ is large, the $\mathcal{L}^{n}$ transform can be approximated by

$$
\begin{array}{r}
\mathcal{L}^{n}\left[e^{-\left(\alpha-x_{g}-u_{g} t\right)^{2}}\right] \simeq \frac{2 k}{\omega_{n}} e^{-k\left(x_{g}+u_{g} t\right)} L_{n}\left(2 k\left(x_{g}+u_{g} t\right)\right) \\
\times \int_{0}^{\infty} e^{-\left(\alpha-x_{g}-u_{g} t\right)^{2}} d \alpha \simeq \\
\frac{2 \sqrt{\pi} k}{\omega_{n}} e^{-k\left(x_{g}+u_{g} t\right)} L_{n}\left(2 k\left(x_{g}+u_{g} t\right)\right) .
\end{array}
$$

Hence, the $n$-th component of the evanescent term Eq. (22) becomes

$\zeta_{g, n}^{e} \simeq \frac{2}{c_{g}} \int_{0}^{\infty} e^{-t v(k)} g_{n}(k) d k$,

where

$$
v(k)=k\left(\frac{x+x_{g}}{t}+u_{g}\right),
$$

and

$$
g_{n}(k)=k e^{-k^{2} /\left(4 c_{g}^{2}\right)} L_{n}(2 k x) L_{n}\left(2 k\left(x_{g}+u_{g} t\right)\right) \cos k y .
$$

Expression Eq. (29) features an integral decaying with time. We shall determine its rate of decay by using the method of asymptotic analysis of integrals devised by Cheng (2007). The dominant contribution to Eq. (29) at large $t$ is given by the point $k$, at which $v(k)$ is minimum, i.e. $k=0$. In a neighbourhood of the dominant point, $g_{n}$ can be Taylor expanded as $g_{n}(k) \simeq k+O\left(k^{2}\right), k \rightarrow 0$. Substituting the expansion for $g_{n}$ into Eq. (29) and then solving the integral, we finally get

$\zeta_{g, n}^{e} \simeq \frac{2}{c_{g}\left(\frac{x+x_{g}}{t}+u_{g}\right)^{2} t^{2}}$

for the leading behaviour of the evanescent term modal components at large time. According to Eq. (30), an observer moving along the $x$ direction at constant speed $x / t$ sees waves decaying all as an $O\left(t^{-2}\right)$. Also, the larger the velocity of the slide $u_{g}$, the faster the decay in the offshore direction of the evanescent waves. The same reasoning as above can be repeated with no significant alterations also for the double parabolic landslide of Eq. (12), so that the relevant rate of decay is

$\zeta_{p, n}^{e} \simeq \frac{32}{9 \pi c_{p}\left(\frac{x+x_{p}}{t}+u_{p}\right)^{2} t^{2}}, \quad \forall y$.

Then the evanescent waves generated by the finite-length, double parabolic landslide have the same rate of decay, $O\left(t^{-2}\right)$, as those generated by the infinitely-long Gaussian landslide. Hence, the behaviour in the near field soon after the generation of the tsunami is not affected significantly by the shape of the slide (as long as the thin slide hypothesis is satisfied and the slide is smooth, see Liu et al., 2005). We next turn to the analysis of the oscillatory components $\zeta_{g}^{o}$ and $\zeta_{p}^{o}$ in the far field.

\subsubsection{Behaviour for large times}

At large times the contribution of evanescent terms to the total wave field can be neglected, so that the free-surface elevation $\zeta$ can be approximated in terms of the oscillatory modes only. For the Gaussian-shaped landslide Eq. (10), the $n$-th 
modal component of the free-surface elevation Eq. (19) evaluated at the shoreline $x=0$ reads then as

$$
\begin{array}{r}
\zeta_{g, n}(0, y, t) \simeq \zeta_{g, n}^{o}(0, y, t)=\frac{2}{\pi} \int_{0}^{\infty}\left[A_{g, n}(k, t) \cos \left(\omega_{n} t\right)\right. \\
\left.+B_{g, n}(k, t) \sin \left(\omega_{n} t\right)\right] \cos (k y) d k
\end{array}
$$

Note that the same formal expression is still valid for the double parabolic landslide Eq. (12), provided the subscript $g$ is replaced by $p$. Careful application of the method of stationary phase (see Sammarco and Renzi, 2008, for details) reveals that the integrand in Eq. (32) admits stationary points at

$$
k_{n}^{*}=(2 n+1)\left(\frac{t}{2 y}\right)^{2}
$$

where the phase function $w_{n}=k y / t-\omega_{n}$ of the products $\cos \left(\omega_{n} t\right) \cos (k y)$ and $\sin \left(\omega_{n} t\right) \cos (k y)$ is null. Near the stationary points of Eq. (33), the latter trigonometric terms experience very slow variations with $k$, so that their phase function can be expanded about $k=k_{n}^{*}$ in a second-order Taylor series: $w_{n}(k) \simeq w_{n}\left(k_{n}^{*}\right)+1 / 2 \ddot{\omega}_{n}\left(k_{n}^{*}\right)\left(k-k_{n}^{*}\right)^{2}$, where $\ddot{\omega}_{n}(k)=-\sqrt{2 n+1} /\left(4 k^{3 / 2}\right)$ is the second derivative of the $n$ th mode eigenfrequency Eq. (8). Then by solving the integral of Eq. (32) with this simplification and summing up all the harmonics, the free-surface elevation at the shoreline can be finally approximated for large times as

$$
\begin{array}{r}
\zeta_{g}(0, y, t) \simeq \sum_{n=0}^{\infty} \zeta_{g, n}^{o}(0, y, t) \simeq \sum_{n=0}^{\infty} \frac{\sqrt{2}}{\left(\pi t\left|\ddot{\omega}_{n}\left(k_{n}^{*}\right)\right|\right)^{1 / 2}} \times \\
\times\left[A_{g, n}\left(k_{n}^{*}\right) \cos \left(k_{n}^{*} y-\omega_{n} t+\frac{\pi}{4}\right)+\right. \\
\left.\quad-B_{g, n}\left(k_{n}^{*}\right) \sin \left(k_{n}^{*} y-\omega_{n} t+\frac{\pi}{4}\right)\right] .
\end{array}
$$

Again, Eq. (34) has been written for the Gaussian-shaped landslide Eq. (10), but it formally holds also for the double parabolic slide Eq. (12) with a simple replacement of the subscript $g$ with $p$. Hence, Eq. (34) describes an oscillatory wave motion propagating along the shoreline. For an observer moving along the $y$ direction at a constant speed of $y / t, k_{n}^{*}$, Eq. (33) is constant and the oscillatory component Eq. (34) decays with time as $O\left(t^{-1 / 2}\right)$, regardless of the shape and boundedness of the slide. Note also that the decay of the transient longshore waves is slower than the decay of the evanescent perturbation moving offshore $\left(O\left(t^{-2}\right)\right)$.

By applying the same method but for $x>0$, we obtain an approximated expression of the free-surface elevation for all $(x, y)$ for large times:

$\zeta_{g}(x, y, t) \approx \sum_{n=0}^{+\infty} e^{-k_{n}^{*} x} L_{n}\left(2 k_{n}^{*} x\right) \zeta_{g, n}^{o}(0, y, t)$,

where again the subscript $g$ is to be replaced with $p$ to obtain the relevant expression for the double parabolic slide. Note the exponential decay in $x$ of the free-surface elevation
Eq. (35): the wave field is now bounded along the coastline and no perturbation is radiated offshore. Hence, no matter the landslide shape and boundedness, at large times after the impact, the energy released by the slide to the water stays trapped along the shoreline. The wave field consists of a system of transient, longshore-travelling edge waves, representing a potential threat for all the near-shore civilian installations. So far, many similarities seem to exist between the wave fields generated by the two model slides. A deeper insight will point out the differences, as shown in the next section.

\subsection{Discussion}

\subsubsection{Experimental comparison}

First, we shall validate the theoretical results of the previous section by comparison with the experimental data of $\mathrm{Di} \mathrm{Ri}-$ sio et al. (2009). The experiments were performed at LIAM (Laboratory of Maritime and Environmental Hydraulics of L'Aquila, Italy) in a basin $5.40 \mathrm{~m}$ long by $10.80 \mathrm{~m}$ wide and $0.8 \mathrm{~m}$ deep. In the experiments an ellipsoidal $0.8 \mathrm{~m}-$ long $(\sigma=0.4 \mathrm{~m})$ by $0.4 \mathrm{~m}$ wide $(\lambda=0.2 \mathrm{~m})$ block is dropped down a steep slope, $s=0.3$, as shown in Fig. 3. The landslide maximum thickness is $0.05 \mathrm{~m}$, and its maximum crosssectional area is about $0.03 \mathrm{~m}^{2}$. The release distance of the ellipsoidal slide, i.e. the distance between the slide front and the mean water level measured upwards along the incline, is set to $X^{\prime}=-0.2 \mathrm{~m}$. In this configuration, the front part of the landslide is submerged at the starting position, but the landslide centroid is outside the water. For the double Gaussian landslide, the shape parameters $\sigma_{g}=0.37 \mathrm{~m}, \eta_{g}=0.045 \mathrm{~m}$ and $c_{g}=\sigma_{g} / \lambda_{g}=2$ are chosen so that the overall area beneath the relevant shape function approximates the experimental landslide maximum cross-sectional area. Following the same criterion for the double parabolic slide, the shape parameters are chosen as $\sigma_{p}=0.44 \mathrm{~m}, \eta_{p}=0.045 \mathrm{~m}$ and $c_{p}=\sigma_{p} / \lambda_{p}=2$. Now let us set the centroid initial position $x_{g}^{\prime}$ and $x_{p}^{\prime}$ for the double Gaussian and the double parabolic slide, respectively, by referring to the laboratory setup of Fig. 3. The centroid initial position $x_{0}^{\prime}$ for the experimental landslide can be obtained from the release distance $X^{\prime}$ by means of the simple geometrical projection

$x_{0}^{\prime}=-\sigma-X^{\prime} \cos \alpha$,

where $\alpha=\arctan s$ is the angle of the incline on the horizontal plane and $\sigma$ the half-length of the slide. By employing the experimental values $X^{\prime}=-0.2 \mathrm{~m}$ and $\sigma=0.4 \mathrm{~m}$ in Eq. (36), we obtain the centroid initial position $x_{0}^{\prime}=-0.18 \mathrm{~m}$. Now, given the centroid initial position, the mean horizontal velocity of the landslide $u_{0}^{\prime}$ needs to be estimated. For this purpose we consider again the experimental results of $\mathrm{Di}$ Risio et al. (2009). By measuring the mean velocity of the slide along the incline $U^{\prime}$ during the underwater motion 
for various release distances $X^{\prime}$ (see Fig. 3), we obtain the empirical linear relation

$U^{\prime}=1.119+1.139 X^{\prime}$,

where the dimensional coefficients $1.119 \mathrm{~m} \mathrm{~s}^{-1}$ and $1.139 \mathrm{~s}^{-1}$ have been derived via the linear regression of the experimental data, as shown in Fig. 4. Let us now express Eq. (37) in terms of the variables $u_{0}^{\prime}$ and $x_{0}^{\prime}$. First assume that $U^{\prime}$ coincides with the landslide average velocity along the incline for all the stages of motion (i.e. subaerial plus submerged); then the velocity $u_{0}^{\prime}$ in the $x^{\prime}$ direction can be obtained from Eq. (37) with the simple geometrical projection

$u_{0}^{\prime}=U^{\prime} \cos \alpha=\left(1.119+1.139 X^{\prime}\right) \cos \alpha$,

where $\alpha=\arctan s$ is still the angle between the incline and the horizontal (see again Fig. 3). Finally, by substituting Eqs. (36) into Eq. (38), the latter can be rewritten as

$u_{0}^{\prime}\left(x_{0}^{\prime}\right)=1.119 \cos \alpha-1.139\left(\sigma+x_{0}^{\prime}\right)$.

This is the sought relation between the landslide horizontal velocity $u_{0}^{\prime}$ and the centroid initial position $x_{0}^{\prime}$, valid for the the experimental setup of Di Risio et al. (2009). Here, we shall take the same values as the experimental ones for the slide initial position Eq. (36) and uniform speed Eq. (39), for both the Gaussian-shaped and the double parabolic slide. Hence, from the values of the centroid initial position $x_{g}^{\prime}=$ $x_{p}^{\prime}=x_{0}^{\prime}=-0.18 \mathrm{~m}$, the values of the average speed $u_{g}^{\gamma}=$ $u_{p}^{\prime}=u_{0}^{\prime}=0.845 \mathrm{~m} \mathrm{~s}^{-1}$ for both slides are derived directly via Eq. (39). In Fig. 5 the time series of the free-surface elevation for the infinite Gaussian-shaped slide Eq. (10) are plotted in physical variables at two different points along the shoreline $x^{\prime}=0$ and compared to the experimental data of Di Risio et al. (2009). Figure 5a shows the time series at $y^{\prime}=3.10 \mathrm{~m}$ away from the point of generation; in Fig. $5 \mathrm{~b}$ is instead depicted the free-surface time series at point $y^{\prime}=4.07 \mathrm{~m}$ from the origin. The main properties of the generated wave field, i.e. the shape of the waves, the time of arrival of crests and troughs and the maximum runup and drawdown, are predicted very satisfactorily by the analytical model. The agreement between analytical and experimental results is good, up to $t^{\prime}=8 \mathrm{~s}$. After that, waves reflected by the side walls in the experiment make the wave field not comparable with that given by the model. In Fig. 5 comparison is also made between the free-surface elevation for the finite double parabolic slide Eq. (12) and the experimental results of Di Risio et al. (2009) at the same points along the shoreline as before. Again, the main physical properties of the wave field are well-reproduced by the model, even if in this case the wave amplitude seems to be slightly overestimated for the larger waves. The excess of mass at the corners of the double parabolic landslide, whose footprint is rectangular and not elliptical as in the experiments, is likely to be the reason for this effect. After having shown a good agreement between the theoretical and the experimental data, in the following sections we analyse the tsunami generation and propagation mechanisms and the parametric dependence of the wave field on the initial position and shape of the slide, enhancing the peculiarities of and the differences between each of the two model slides considered so far.

\subsubsection{Tsunami generation and propagation}

The three-dimensional dynamics of tsunami generation and propagation along the indefinite plane beach lying on the $y^{\prime}$ axis can be appreciated with the plots of Fig. 6. Here, threedimensional snapshots of the free-surface elevation are presented at fixed times, starting from the beginning of the motion till the perturbation is fully generated and propagating away from the source. The double Gaussian landslide with parameters $\sigma_{g}=0.37 \mathrm{~m}, \eta_{g}=0.045 \mathrm{~m}, c_{g}=2, x_{g}^{\prime}=0 \mathrm{~m}$ and $u_{g}^{\prime}=1 \mathrm{~m} \mathrm{~s}^{-1}$ is taken as a reference model. At the earliest times of motion, the landslide pushes water ahead, while a depression generates just landwards of the slide (Fig. 6a, b). Hence, large free-surface gradients are created along the shoreline, driving strong fluxes to converge towards the centre (Fig. 6c). As a result, the convergent flows eventually collide at the origin to form a large elevation wave. Finally, the rebound wave splits into two crests, which symmetrically start to travel along the shoreline (Fig. 6d) and the wave field is fully developed. After having highlighted the dynamics of tsunami generation, in the following sections we shall consider the dependence of the generated wave field on the main parameters of the problem, namely the initial position of the slide and its shape.

\subsubsection{Parametric analysis: the slide initial position}

Figure 7 shows the behaviour of the maximum free-surface elevation of the first (dashed line) and second (solid line) incoming waves versus the centroid initial position $x_{g}^{\prime}$, at a point along the shoreline $\left(y^{\prime}=3.10 \mathrm{~m}\right)$ for the reference double Gaussian slide defined above. The induced waves reach their maximum amplitude when the slide is fully subaerial and the corresponding velocity (see Eq. 39) is larger than $1 \mathrm{~m} \mathrm{~s}^{-1}$ (see Fig. 4). Hence, the higher the slide initial position on the incline, the larger the amplitude of the induced wave field at a point on the shoreline far from the slide. However, increased dissipations due to viscosity and vortices generated at the impact, not taken into account in this model, would certainly reduce the amplitude of the generated waves below the theoretical values. Moving the landslide towards the origin both increases $x_{g}^{\prime}$ and reduces $u_{g}^{\prime}$. As a result, the amplitudes of the generated waves decay quickly, due to the reduced exchange of total energy between the slide and the fluid (see again Fig. 7). Let us now investigate the influence of the shape of the slide on the generated wave field. 


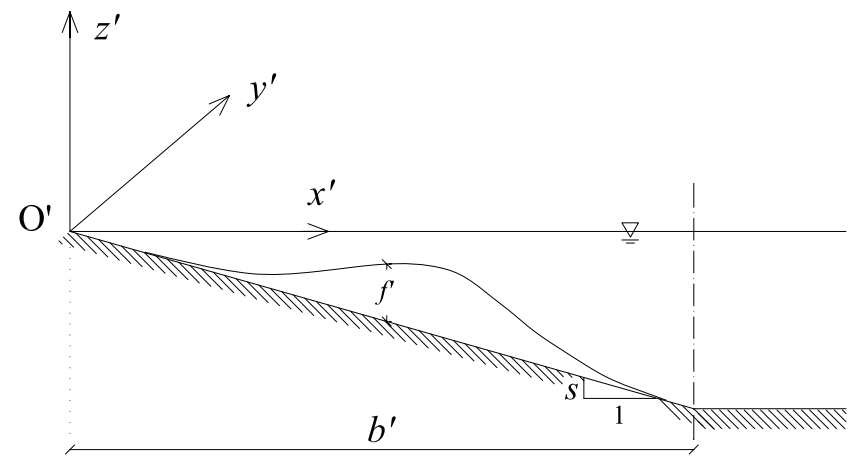

Fig. 9. The fluid domain of the semi-plane beach in physical coordinates. The dashed line divides the near field $x^{\prime}<b^{\prime}$ from the far field $x^{\prime}>b^{\prime}$.

\subsubsection{Parametric analysis: the shape of the slide}

In Fig. 8 the free-surface time series for both the infinite Gaussian-shaped slide Eq. (10) and the finite double parabolic slide Eq. (12) are plotted at the same points, along the shoreline, i.e. $y^{\prime}=3.10 \mathrm{~m}$ (Fig. 8a) and $y^{\prime}=4.07 \mathrm{~m}$ (Fig. 8b) for the geometry of Sect. 2.4.1. The general behaviour of both curves is very similar, with the largest wave shifted towards the middle of the incoming wave group, a typical feature of landslide generated tsunamis (see Lynett and Liu, 2005; Sammarco and Renzi, 2008). This behaviour, independent of the shape and dimensions of the slide, can be explained theoretically as follows (see Gonzalez et al., 1995, for a similar argument). Back to Sect. 2, the phase velocity of the individual wave modes is $c_{n}=\omega_{n} / k=$ $\sqrt{(2 n+1) / k}$, while the group velocity is $C g_{n}=d \omega_{n} / d k=$ $\sqrt{(2 n+1) / k} / 2$, so that $C g_{n}=c_{n} / 2$. The difference between phase and group velocity explains the dispersive behaviour of the edge waves propagating along the beach at large times, already noticed numerically by Lynett and Liu (2005) and Bellotti et al. (2008) and analytically by Sammarco and Renzi (2008). When the landslide moves into water, the initial disturbance of the free-surface evolves into groups of edge waves, each group travelling at velocity $C g_{n}$ along the shoreline. In turn, the single wave crests propagate inside the group at a larger speed $c_{n}$. Since the group velocity is the velocity of the energy transport, we expect the bulk of the energy released by the slide into the water to travel at a lower speed $C g_{n}$ than that of the first generated wave crests $c_{n}$. This explains why, regardless of the geometry of the landslide, the larger waves, which are also the most energetic ones, are always shifted towards the middle of the group. Note that the linear long wave theory, non-dispersive over bottoms of constant depth, reproduces the dispersive behaviour of the trapped waves over inclined bottoms. Despite a general similarity between the wave fields generated by the Gaussian-shaped slide and the double parabolic slide, some differences can be pointed out. The Gaussian-shaped

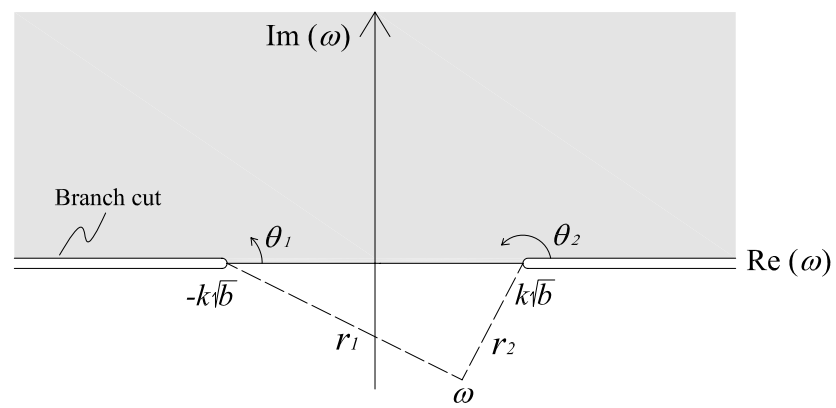

Fig. 10. Branch cuts in the complex plane for $\beta(k, \omega)$ Eq. (55). The complex frequency $\omega \in \Omega$, which is the unshaded area corresponding to the lower half-plane, real axis included. Angles $\theta_{1}$ and $\theta_{2}$ are measured counterclockwise from the real axis on the chosen Riemann sheet Eq. (58).

slide has infinite length and width, which is a limit of the SR model and the reason that motivated this work. Furthermore, the Gaussian is smoother than the double paraboloid, having no abrupt terminations at the sides (see Fig. 2). These two specificities can account for the dissimilarities between both models (see again Fig. 8), such as the difference in wave amplitudes and the accentuation of spiky, double-crested waves occurring with the double parabolic slide. Mathematically, this is a side effect of the wave Eq. (3), which propagates the irregularities (i.e. the cuts) of the forcing function Eq. (12) without smoothing them completely. Physically, these double crested waves can be explained with the secondary inertial rebound generated at the origin soon after the tail of the slide enters water, as shown by Lynett and Liu (2005) and Sammarco and Renzi (2008). Hence, this effect is mostly enhanced for landslides whose tail terminates abruptly, like the double parabolic one, rather than for smooth infinite bodies like the Gaussian slide. In conclusion, the finiteness of the landslide is an important feature that cannot be neglected in analytical models, aiming to reproduce accurately the shape of the generated waves. Having investigated the effect of the slide shape and finiteness, in the next section we move into the analysis of the influence of the continental platform on landslide-generated tsunamis.

\section{Influence of the continental platform}

\subsection{Position of the problem}

In Sect. 2 we have shown that, within the linear shallowwater theory, the landslide-forced waves over an indefinite incline rapidly decay in the offshore direction, while a transient system of longshore travelling edge waves propagates away from the landslide. However, an infinite slope does not fully represent a coastal area. To obtain a more realistic model, in this section we investigate a different geometry, 


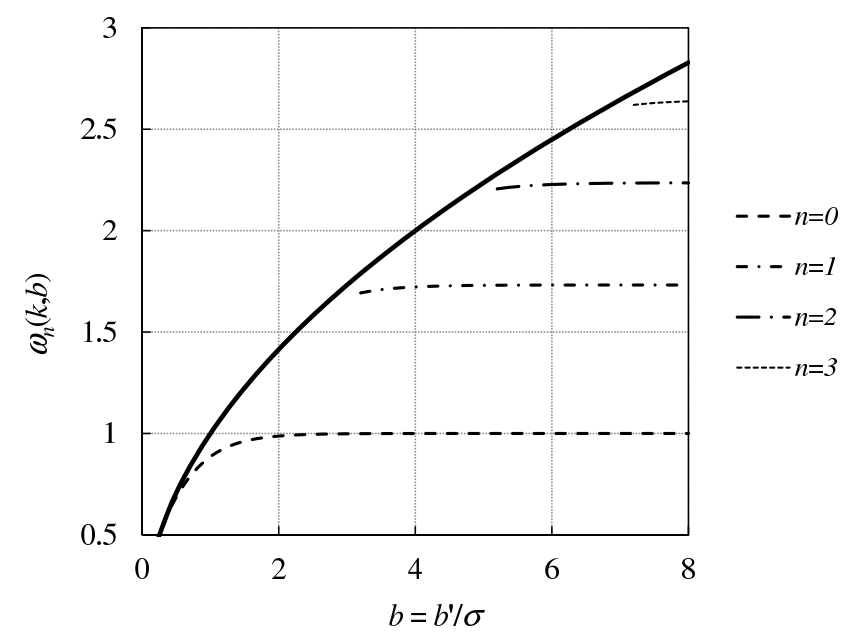

Fig. 11. Behaviour of the first eigenvalues $\omega_{n}, n=0,1,2,3$, versus $b$ for $k=1$. The bold line represents the upper limit $\omega=k \sqrt{b}$ of the domain Eq. (85).

where the slope extends to a finite length and further connects to a continental platform of constant depth. The physical domain of the model is shown in Fig. 9. Having set a 3 -D system of coordinates $\left(x^{\prime}, y^{\prime}, z^{\prime}\right)$, water is in the region $x^{\prime}>0$. At a distance $b^{\prime}$ from the coastline, the slope ends and connects to a flat bottom of constant depth $h_{b^{\prime}}^{\prime}$. Due to the geometric discontinuity introduced by the flat-bottom zone, we shall define the near field as the domain $x^{\prime}<b^{\prime}$, where $b^{\prime}$ is the horizontal length of the incline. As a consequence, we shall also define the far field as the region $x^{\prime}>b^{\prime}$, where the bottom depth is constant $h^{\prime}=h_{b^{\prime}}^{\prime}=s b^{\prime}, s$ being the slope of the incline. The system is assumed to be symmetric with respect to the $y^{\prime}$ axis; also assume that the landslide motion starts in a neighbourhood of the origin and that the landslide shape is symmetric with respect to $y^{\prime}$. Within the assumption of linear shallow-water waves, the behaviour of the fluid is still described by the forced long-wave Eq. (1), where the bottom depth $h^{\prime}$ is now defined as

$h^{\prime}\left(x^{\prime}<b^{\prime}\right)=s x^{\prime}, \quad h^{\prime}\left(x^{\prime} \geq b^{\prime}\right)=h_{b^{\prime}}^{\prime}=s b^{\prime}$,

according to the geometry of Fig. 9. By introducing the same non-dimensional variables of Eq. (2), the equation of motion Eq. (1) becomes

$h \zeta_{x x}+h_{x} \zeta_{x}+h \zeta_{y y}=\zeta_{t t}-f_{t t}$.

In Eq. (41) $h=h^{\prime} / \sigma s$ is the non-dimensional bottom depth

$h(x<b)=x, \quad h(x \geq b)=h_{b}=b$,

where $b=b^{\prime} / \sigma$ is the non-dimensional horizontal beach length. As already done in Sect. 2 for the plane beach, we require the free-surface elevation to be bounded at the shoreline and as $x \rightarrow \infty$ and also to be an even function of the shoreline coordinate $y$. Finally, we require null initial free-surface elevation and velocity, i.e. $\zeta(x, y, 0)=0, \zeta_{t}(x, y, 0)=0$.

\subsection{Solution}

By employing the cosine Fourier transform Eq. (4), the equation of motion Eq. (41) becomes

$h \hat{\zeta}_{x x}+h_{x} \hat{\zeta}_{x}-k^{2} h \hat{\zeta}=\hat{\zeta}_{t t}-\hat{f}_{t t}(x, k, t)$.

Recall from Sect. 2 that, for the simple plane-beach geometry, the system eigenfunctions are the Laguerre polynomials $L_{n}$, with the relevant eigenvalues $\omega_{n}$ given by Eq. (8). To determine the eigenfunctions and the eigenvalues of the semi-plane beach geometry, we seek the solution $\hat{\zeta}_{h}$ of the homogeneous equation associated with Eq. (43):

$h \hat{\zeta}_{x x}+h_{x} \hat{\zeta}_{x}-k^{2} h \hat{\zeta}-\hat{\zeta}_{t t}=0$

By introducing the further transformations

$\xi=2 k x, \quad \hat{\zeta}=e^{-\xi / 2} Z(\xi, k, t)$.

Equation (44) becomes

$4 k^{2}\left[h Z_{\xi \xi}+\left(h_{\xi}-h\right) Z_{\xi}-\frac{1}{2} h_{\xi} Z\right]-Z_{t t}=0$.

To solve Eq. (46) we shall assume the following separation of variables for $Z(\xi, k, t)$ :

$Z=\Re\left\{X(\xi) e^{-\mathrm{i} \omega t}\right\}$

where $X(\xi)$ is the unknown spatial part of the homogeneous solution, and $\omega$ a complex wave frequency. Note that the complex frequency $\omega=\omega_{r}+\mathrm{i} \omega_{i}$ must have a null or negative imaginary part, $\omega_{i} \leq 0$ in order for Eq. (47) not to diverge for large $t$, i.e. $\omega \in \Omega$, where $\Omega=\{\omega \in \mathbb{C}:-\pi \leq \arg \omega \leq 0\}$ is the lower complex half plane, real axis included. With the substitution dictated by Eq. (47), Eq. (46) can be finally rewritten as

$h X_{\xi \xi}+\left(h_{\xi}-h\right) X_{\xi}-\left(\frac{h_{\xi}}{2}-\frac{\omega^{2}}{4 k^{2}}\right) X=0$.

The latter is to be solved separately in the two fields; the two solutions obtained will be matched afterwards at the common boundary to assure continuity of pressure and fluxes.

In the near field $h=x$ or, in terms of the transformed variables of Eq. (45), $h=\xi / 2 k$. By using the latter expression, the homogeneous form of the governing Eq. (48) becomes

$\xi X_{\xi \xi}+(1-\xi) X_{\xi}-\alpha X=0$,

where

$\alpha=\alpha(k, \omega)=1 / 2\left(1-\omega^{2} / k\right)$.

Equation (49) is the Kummer's equation of parameter $\alpha$, whose general solution is a linear combination of the Kummer functions of first and second kind (see Abramowitz and Stegun, 1972), respectively $M$ and $U$, i.e. $X(\xi, k)=$ 

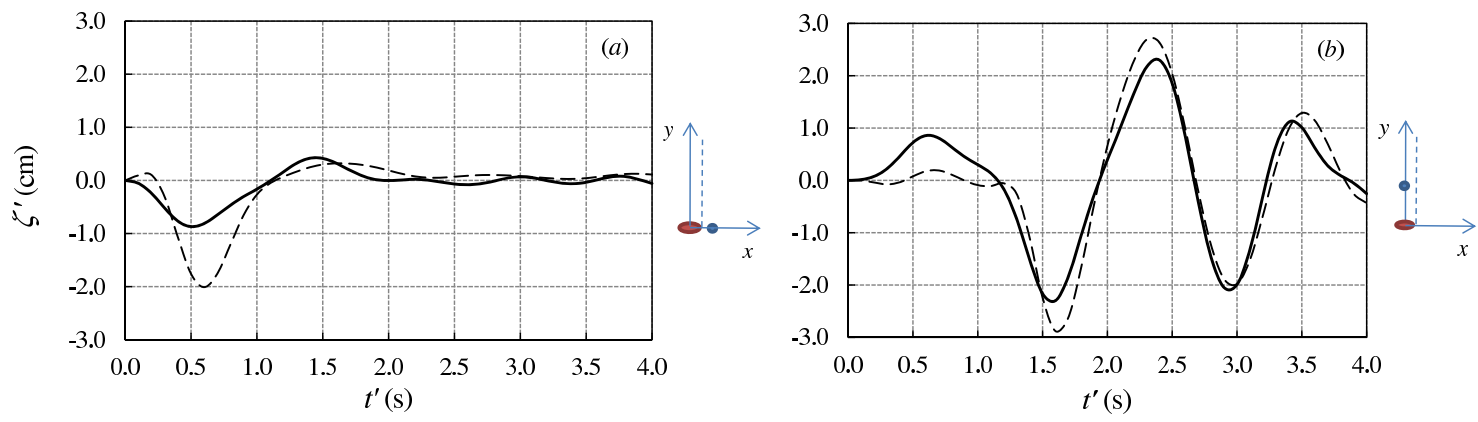

Fig. 12. Time series of the free-surface elevation in physical variables at points: $(\mathbf{a}):\left(x^{\prime}, y^{\prime}\right)=(0.4,0) \mathrm{m}$, and $(\mathbf{b}):\left(x^{\prime}, y^{\prime}\right)=(0,0.8) \mathrm{m}$. Solid lines (-) represent the time series for the semi-plane beach of horizontal length $b=b^{\prime} / \sigma=1$, while dashed lines (- -) are relevant to the indefinite plane beach model.
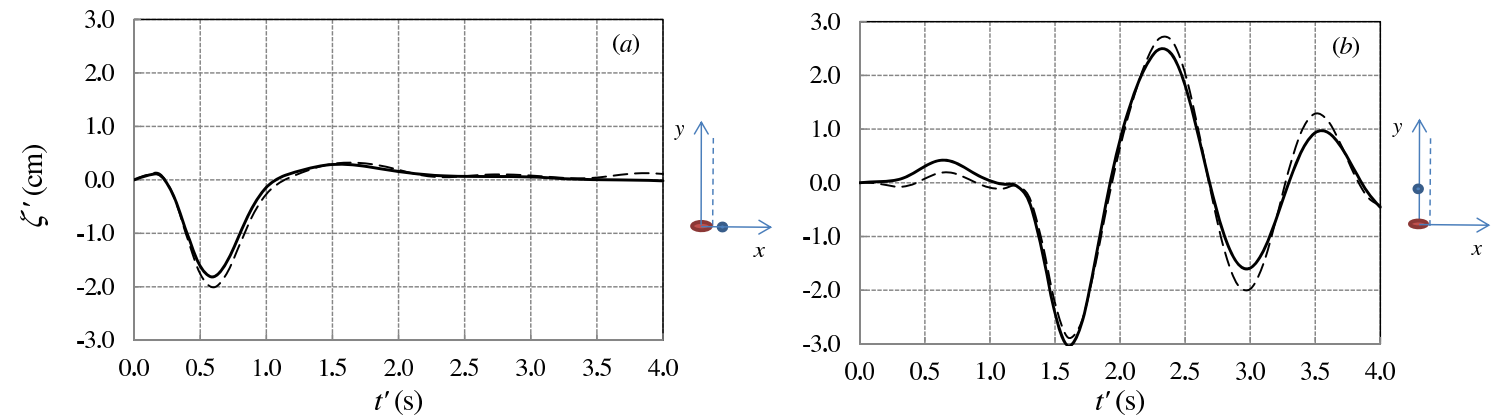

Fig. 13. Time series of the free-surface elevation in physical variables at points: (a): $\left(x^{\prime}, y^{\prime}\right)=(0.4,0) \mathrm{m}$ and $(\mathbf{b}):\left(x^{\prime}, y^{\prime}\right)=(0,0.8) \mathrm{m}$. Solid lines (-) represent the time series for the semi-plane beach of horizontal length $b=10$, while dashed lines (- -) are relevant to the indefinite plane-beach model. Note that for $b \gg 1$ the two models converge to the same results.

$A M(\xi, \alpha)+B U(\xi, \alpha)$, where $A$ and $B$ are integration constants. Since the Kummer function of second kind has a logarithmic singularity at the origin, boundedness of the solution at the shoreline requires $B=0$, i.e.

$X(\xi, k)=A M(\xi, \alpha), \quad \xi<2 k b$.

Hence, the homogeneous solution of Eq. (46) in the near field is simply $Z_{h}(\xi, k, t)=X(\xi, k) e^{-\mathrm{i} \omega t}=A M(\xi, \alpha) e^{-\mathrm{i} \omega t}$, $\Re$ having been omitted for brevity, and in terms of the original variables of Eq. (45)

$\hat{\zeta}_{h}(x, k, t)=A e^{-k x} M(2 k x, \alpha) e^{-\mathrm{i} \omega t}, \quad x<b$.

In the far field $h=b$, so that the homogeneous form of the governing Eq. (48) becomes

$X_{\xi \xi}-X_{\xi}+\frac{\omega^{2}}{4 b k^{2}} X=0$.

This is also a linear ODE of the second order, whose general solution is

$X(\xi, k)=C e^{(1-\beta) \xi / 2}+D e^{(1+\beta) \xi / 2}$,

with $C$ and $D$ integration constants and

$\beta=\beta(k, \omega)=\frac{1}{k \sqrt{b}}(k \sqrt{b}-\omega)^{1 / 2}(k \sqrt{b}+\omega)^{1 / 2}$, a complex parameter. The solution of the homogeneous Eq. (44) in the far field is then obtained by substituting the spatial component $X(\xi, k)$ into the general form Eq. (47):

$\hat{\zeta}_{h}(x, k, t)=C e^{-\beta k x-\mathrm{i} \omega t}+D e^{\beta k x-\mathrm{i} \omega t}, \quad x>b$,

where usage of Eq. (45) has also been made to switch back to the original variables. In Eq. (56) the complex parameter $\beta$ Eq. (55), is given by the product of two square roots of complex variable and admits two branch points in the complex domain $\Omega$, respectively at $\omega=\mp k \sqrt{b}$, i.e. the zeros of the square roots. As a consequence, we introduce two semiinfinite branch cuts along the real axis of $\Omega$, as shown in Fig. 10 (see Mei, 1997, for a similar example). To evaluate $\beta$ correctly on the different edges of the cuts of Fig. 10, let us define

$\omega+k \sqrt{b}=r_{1} e^{\mathrm{i} \theta_{1}}, \quad k \sqrt{b}-\omega=r_{2} e^{\mathrm{i}\left(\theta_{2}-\pi\right)}$,

where the phase angles $\theta_{1,2}$ are measured counterclockwise from the positive real axis. To avoid multivaluedness of the square roots in Eq. (55), let us first consider the Riemann sheet defined by $\theta_{1} \in[-\pi, \pi), \theta_{2} \in(0,2 \pi]$. Then restrict the range of variation of $\theta_{1,2}$ by requiring $\omega \in \Omega$ (unshaded region of Fig. 10, including the real axis), so that

$\theta_{1} \in[-\pi, 0], \quad \theta_{2} \in[\pi, 2 \pi]$. 
Note that, according to Eq. (58), $\theta_{1}=-\pi$ describes the lower edge of the left-hand cut on the negative real axis, while $\theta_{2}=2 \pi$ describes the lower edge of the right-hand cut on the positive real axis. Other values of $\theta_{1,2}$ in the range defined by Eq. (58) correspond to points in the lower complex plane. Substituting Eq. (57) into Eq. (55), we obtain for the complex parameter

$\beta=\sqrt{\frac{r_{1} r_{2}}{b k^{2}}} e^{\mathrm{i}\left(\theta_{1}+\theta_{2}-\pi\right) / 2}$.

Now, if the frequency $\omega$ is anywhere along the the lower edge of the right-hand cut, then $\theta_{1}=0$ and $\theta_{2}=2 \pi$, so that $\beta=\mathrm{i}|\beta|$ is imaginary with positive sign. A similar analysis can be done also for the left-hand cut, where $\beta=-\mathrm{i}|\beta|$ on the lower edge. In all these cases the corresponding freesurface elevation in the far field Eq. (56) is an oscillating function of the offshore coordinate $x$ and physically represents incoming and outgoing progressive waves. Finally, if $\omega$ is on the real axis between $-k \sqrt{b}<\omega<k \sqrt{b}$, then $\theta_{1}=0$ and $\theta_{2}=\pi$, so that Eq. (59) gives $\beta=\sqrt{r_{1} r_{2}} / k \sqrt{b}$, i.e. a positive real number. As a consequence, the second term of the far field solution Eq. (56) would grow without boundaries as $x \rightarrow \infty$ unless $D=0$. With this position the homogeneous solution in the far field Eq. (56) becomes

$\hat{\zeta}_{h}(x, k, t)=C e^{-\beta k x-\mathrm{i} \omega t}, \quad x>b$,

while the relevant spatial component $X$ Eq. (54) reduces to

$X(\xi, k)=C e^{(1-\beta) \xi / 2}, \quad \xi>2 k b$.

\subsubsection{Matching and eigenvalues}

The solutions of the homogeneous Eq. (44) in the near field and in the far field, Eqs. (52) and (60) respectively, must now be matched at the common boundary $x=b$. Continuity of the free-surface elevation $\zeta$ and the fluxes $\partial \zeta / \partial x$ yields, respectively,

$$
\begin{gathered}
A e^{-k b} M(2 k b, \alpha)=C e^{-\beta k b} \\
A e^{-k b}\left[-k M(2 k b, \alpha)+M_{x}(2 k b, \alpha)\right]=-C \beta k e^{-\beta k b} .
\end{gathered}
$$

This homogeneous system with unknowns $A$ and $C$ admits a non-trivial solution $(A, C) \neq(0,0)$ if and only if

$\Delta(k, \omega)=M_{x}(2 k b, \alpha)+k(\beta-1) M(2 k b, \alpha)=0$,

where $\Delta \in \mathbb{C}, k>0$ is a real parameter and $\alpha(\omega)$ and $\beta(\omega)$ are given by Eqs. (50) and (55), respectively. Equation (63) represents the eigenvalue condition of the matching system Eq. (62); the complex values $\omega_{n}$, for which Eq. (63) is satisfied, are the sought eigenvalues. Numerical solution of Eq. (63) shows that, for a given value of $k$, there is an enumerable set of $2 \cdot[N(k, b)+1]$ real eigenvalues $\pm \omega_{0}, \pm \omega_{1} \ldots \pm \omega_{n} \ldots \pm \omega_{N}$ on the chosen Riemann sheet, such that $-k \sqrt{b}<-\omega_{N}<\ldots<-\omega_{0}<0<\omega_{0}<\ldots<\omega_{N}<$ $k \sqrt{b}$, i.e. $\pm \omega_{n} \in(-k \sqrt{b}, k \sqrt{b}), \quad n=0,1, \ldots, N(k, b) \in \mathbb{N}$.
When $\omega= \pm \omega_{n}$, the solution of the system Eq. (62) is straightforward:

$$
C=M(2 k b, \alpha) e^{(\beta-1) k b},
$$

having set the arbitrary parameter $A=1$. Now consider the spatial eigenfunctions in the near and far field given respectively by Eqs. (51) and (61), with $\omega=\omega_{n}$ :

$$
\begin{array}{rr}
X_{n}(\xi, k)=M\left(\xi, \alpha_{n}\right) & \text { if } \xi<2 k b \\
X_{n}(\xi, k)=M\left(2 k b, \alpha_{n}\right) e^{\left(1-\beta_{n}\right)(\xi / 2-k b)} & \text { if } \xi \geq 2 k b,
\end{array}
$$

with $n=0,1, \ldots, N(k, b)$, where the Kummer function

$M\left(\xi, \alpha_{n}\right)=\sum_{m=0}^{\infty} a_{n m}(\omega) \xi^{m}$,

and $a_{n m}=\left(\alpha_{n}\right)_{m} /(m !)^{2}$, being $\left(\alpha_{n}\right)_{m}=\alpha_{n}\left(\alpha_{n}+1\right)\left(\alpha_{n}+\right.$ 2)...( $\left.\alpha_{n}+m-1\right)$ (see again Abramowitz and Stegun, 1972). Finally, in Eq. (64)

$\alpha_{n}=\alpha\left(k, \omega_{n}\right)=\frac{1}{2}-\frac{\omega_{n}^{2}}{2 k}, \beta_{n}=\beta\left(k, \omega_{n}\right)=\sqrt{1-\frac{\omega_{n}^{2}}{b k^{2}}}$,

and again $\xi=2 k x$. Note that for a given modal order $n$, both the real eigenvalues $\pm \omega_{n}$ correspond to the same real parameters $\alpha_{n}$ and $\beta_{n}$ Eq. (66), i.e. both $\pm \omega_{n}$ are relevant to the same spatial eigenfunction Eq. (64). Therefore, to ensure uniqueness of the solution, we retain only the eigenmodes associated to the positive eigenvalues $\omega_{n} \in(0, k \sqrt{b})$. Referring back to Eqs. (45) and (47), the solution of the homogeneous Eq. (48) can be written as the linear superposition of all the $(N+1)$ modes

$\hat{\zeta}_{h}(x, k, t)=\sum_{n=0}^{N(k)} e^{-k x} X_{n}(2 k x) e^{-\mathrm{i} \omega_{n} t}$,

where the $X_{n}$ s are given by Eq. (64). To investigate the physical nature of the perturbation described by Eq. (67), let us consider the spatial part:

$\hat{\eta}_{n}(x, k, t)=e^{-k x} X_{n}(2 k x)$

of the $n$-th modal component of the free-surface elevation $\hat{\zeta}_{h}(x, k, t)$. Using Eq. (64) to express the eigenfunctions $X_{n}$, Eq. (68) becomes

$\hat{\eta}_{n}(x, k, t)=M\left(2 k x, \alpha_{n}\right) e^{-k x}$

in the near field $x<b$ and

$\hat{\eta}_{n}(x, k, t)=M\left(2 k b, \alpha_{n}\right) e^{-k b\left(1-\beta_{n}\right)} e^{-\beta_{n} k x}$,

in the far field $x \geq b$. Since the eigenvalues $\omega_{n}$ are all real and positive, with $\omega_{n}<k \sqrt{b}$, the parameter $\beta_{n}$ Eq. (66) is a positive real number too. As a consequence, all the spatial components $\hat{\eta}_{n}$ of the free-surface elevation, proportional to $e^{-\beta_{n} k x}$ in the far field (see Eq. 70), decay exponentially while 
moving in the offshore direction at a large distance from the shore. Hence, all the natural eigenmodes of the semi-plane beach are trapped along the shoreline and no wave propagates to infinity, even if the presence of the flat-bottom region would suggest a propagating nature of the perturbation. This somehow unexpected fact, i.e. that long waves existing at a slope discontinuity are unable to propagate towards larger distances, has been already pointed out also by Longuet-Higgins (1967) and Mei et al. (2005). Physically, trapped waves can be excited linearly only by a localised perturbation at the coastline, e.g. a landslide. Since such a perturbation moves only a finite volume of water in a finite amount of time, the generated edge-wave spectrum involves no radiation to infinity (Meyer, 1971). However, this does not exclude that in cases where nonlinearity is dominant, e.g. when the slide is thick, resonant amplification of the offshore-going wave can occur, making it comparable to the oscillations along the shoreline (Liu et al., 2003).

\subsection{Wave field}

After having investigated the homogeneous problem Eq. (44), we now seek the solution of the forced equation of motion Eq. (43). By using the transformations dictated by Eq. (45), Eq. (43) becomes

$$
\begin{array}{r}
4 k^{2}\left[h Z_{\xi \xi}+\left(h_{\xi}-h\right) Z_{\xi}-1 / 2 h_{\xi} Z\right]-Z_{t t} \\
=-e^{-\xi / 2} \hat{f}_{t t}(\xi / 2, k, t) .
\end{array}
$$

To solve the latter equation, we shall employ the method of variation of parameters, assuming for $Z(\xi, k, t)$ the following expression:

$Z(\xi, k, t)=\sum_{n=0}^{N(k)} X_{n}(\xi) T_{n}(k, t)$

where $X_{n}$ Eq. (64) are the spatial eigenfunctions of the associated homogeneous problem Eq. (48) and $T_{n}$ are unknown functions to be determined. Now substitute the series expansion Eq. (72) into the governing Eq. (71) and exploit the orthogonality property Eq. (A9) of the homogeneous eigensolutions $X_{n}$, thus, getting

$T_{n, t t}+\omega_{n}^{2} T_{n}=\frac{1}{\chi_{n}^{2}} \int_{0}^{\infty} e^{-\xi} X_{n}(\xi) \hat{f}_{t t}(\xi / 2, k, t) e^{-\xi / 2} d \xi$

for the unknown functions $T_{n}$. In Eq. (73), $\omega_{n}$ are still the eigenvalues of the associated homogeneous problem and $\chi_{n}$ Eq. (A8) is the square norm of the relevant eigenfunctions Eq. (64); finally, $\hat{f}$ is the cosine Fourier transform of the landslide shape function. The solution of Eq. (73) is straightforward:

$T_{n}(k, t)=\frac{2 k}{\omega_{n} \chi_{n}^{2}} \int_{0}^{\infty} e^{-k \rho} X_{n}(2 k \rho) I_{n}(\rho, k, t) d \rho$,

where $\rho=\xi / 2 k$ and $I_{n}(\rho, k, t)$ is the same expression as Eq. (7) for the indefinite plane beach. Again, $I_{n}$ can be determined only once the landslide shape function $f$ is defined.
Finally, inverse transform of Eq. (72) together with the substitutions dictated by Eq. (45) yield the free-surface elevation

$\zeta(x, y, t)=\frac{2}{\pi} \int_{0}^{\infty} \sum_{n=0}^{N(k)} X_{n}(2 k x) T_{n}(k, t) \cos k y d k$,

where the $T_{n}$ are given by Eq. (74) and $X_{n}$ Eq. (64) are the eigenfunctions of the associated homogeneous problem Eq. (46). Further analysis can be done only after the forcing function $f(x, y, t)$ is specified.

\subsubsection{Tsunami generated by a double Gaussian-shaped landslide}

In this section we determine the analytical form of the freesurface elevation Eq. (75) generated by a landslide whose shape function is

$f(x, y, t)=\exp \left[-\left(x-x_{0}-u_{0} t\right)^{2}\right] s(y) H(b-x)$

where $s(y)=e^{-(c y)^{2}}$ is still the lateral spreading function and $c=\sigma / \lambda$ the landslide shape coefficient. Equation (76) represents a double Gaussian-shaped landslide sliding along the incline with uniform velocity $u_{0}$ from the initial position $x_{0}$. With the position of Eq. (76) $f$ is non-zero only in the near field $x<b$, while in the far field $x>b$ the direct influence of the forcing term on the generated perturbation is assumed to be negligible, i.e. $f \simeq 0$. Since the effects of the parameters $x_{0}$ and $u_{0}$ on the generated wave field have been already investigated in Sect. 2, in this section we shall limit our analysis to the case $x_{0}=0$ and $u_{0}=1$, describing a halfsubmerged landslide moving with unit horizontal velocity. This assumption simplifies the algebra, yet allows us to investigate the influence of the beach horizontal length $b$ upon the generated wave field. Substituting Eq. (76) with $x_{0}=0$ and $u_{0}=1$ into $I_{n}(\rho, k, t)$, the latter becomes

$$
\begin{aligned}
& I_{n}=\omega_{n} \hat{s}(k)\left\{\left[\omega_{n} a_{n}-e^{-\rho^{2}}\right] \cos \omega_{n} t\right. \\
& \left.-\left[\frac{2 \rho}{\omega_{n}} e^{-\rho^{2}}+\omega_{n} b_{n}\right] \sin \omega_{n} t+e^{-\rho^{2}}\right\},
\end{aligned}
$$

with $\rho<b$, where $\hat{s}(k)=\sqrt{\pi} /(2 c) e^{-k^{2} / 4 c^{2}}$ is the cosine Fourier transform of the spreading function $s(y)$ and

$$
\begin{array}{r}
a_{n}(\rho, k, t)=\frac{\sqrt{\pi}}{2} e^{-\omega_{n}^{2} / 4} \mathfrak{\Im}\left\{e ^ { \mathrm { i } \omega _ { n } \rho } \left[\operatorname{erf}\left(\rho+\mathrm{i} \frac{\omega_{n}}{2}\right)\right.\right. \\
\left.\left.-\operatorname{erf}\left(\rho-t+\mathrm{i} \frac{\omega_{n}}{2}\right)\right]\right\},
\end{array}
$$

while

$$
\begin{aligned}
& b_{n}(\rho, k, t)=\frac{\sqrt{\pi}}{2} e^{-\omega_{n}^{2} / 4} \Re\left\{e ^ { \mathrm { i } \omega _ { n } \rho } \left[\operatorname{erf}\left(\rho+\mathrm{i} \frac{\omega_{n}}{2}\right)\right.\right. \\
&\left.\left.-\operatorname{erf}\left(\rho-t+\mathrm{i} \frac{\omega_{n}}{2}\right)\right]\right\} .
\end{aligned}
$$

Substituting Eq. (77) into $T_{n}$ Eq. (74) and then the latter into Eq. (75), we finally obtain the analytical form of the freesurface elevation $\zeta(x, y, t)$. Let us now follow the same steps 
of Sect. 2 to investigate the physical meaning of Eq. (75). First, define the integral transform $\mathcal{M}^{n}$ of a given function $u(\rho, k, t)$ :

$\mathcal{M}^{n}[u](k, t)=\frac{2 k}{\omega_{n} \chi_{n}^{2}} \int_{0}^{b} e^{-k \rho} M\left(2 k \rho, \alpha_{n}\right) u(\rho, k, t) d \rho$,

where $M$ is still the Kummer function of first kind and $\alpha_{n}$ is given by Eq. (66). Then, by making use of Eq. (80), rewrite Eq. (74) as $T_{n}=\mathcal{M}^{n}\left[I_{n}\right]$. Hence, by simply applying the transform $\mathcal{M}^{n}$ to Eq. (75), we can formally write again $\zeta=\zeta^{o}+\zeta^{e}$, where

$$
\begin{array}{r}
\zeta^{o}=\frac{2}{\pi} \int_{0}^{\infty} \sum_{n=0}^{N(k)} e^{-k x} X_{n}(2 k x)\left[A_{n} \cos \omega_{n} t\right. \\
\left.+B_{n} \sin \omega_{n} t\right] \cos k y d k,
\end{array}
$$

with

$$
\begin{aligned}
& A_{n}=A_{n}(k, t)=\omega_{n} \hat{s} \mathcal{M}^{n}\left[\omega_{n} a_{n}(\rho, k, t)-e^{-\rho^{2}}\right], \\
& B_{n}=B_{n}(k, t)=-\hat{s} \mathcal{M}^{n}\left[2 \rho e^{-\rho^{2}}+\omega_{n}^{2} b_{n}(\rho, k, t)\right],
\end{aligned}
$$

and

$$
\begin{aligned}
\zeta^{e}=\frac{2}{\pi} & \int_{0}^{\infty} \sum_{n=0}^{N(k)} e^{-k x} X_{n}(2 k x) \omega_{n} \hat{s} \\
& \times \mathcal{M}^{n}\left[e^{-(\rho-t)^{2}}\right] \cos k y d k,
\end{aligned}
$$

$X_{n}$ being the eigenfunctions of Eq. (64). Now, $\zeta^{o}$ Eq. (81) describes an oscillatory motion in time. The coefficients $A_{n}$ and $B_{n}$ are formally similar to those already found in Sect. 2 for the indefinite plane beach. The differences lie in three aspects: (1) the usage of the Kummer transform $\mathcal{M}^{n}$ instead of the Laguerre one, $\mathcal{L}^{n},(2)$ the presence of the eigenfunctions $X_{n}$ instead of the Laguerre polynomials, $L_{n}$ and finally (3) the truncation of the sum to the order $N(k) . \zeta^{e}$ is an evanescent component decaying with time, its expression being similar to the evanescent term of the indefinite plane beach of Sect. 2. Again, the landslide generates a twofold wave field made up by oscillatory and evanescent components, the latter rapidly vanishing with time. Due to the analytical similarity between the wave field of the semi-plane beach and that of the indefinite plane beach, all the observations already made in Sect. 2 can be repeated also for the current system. In the following we shall focus our attention on the influence of the main system parameter, i.e. the non-dimensional horizontal length of the incline $b$, on the behaviour of the generated wave field.

\subsection{Discussion: influence of $b$}

As a first step to investigate the influence of the beach nondimensional horizontal length $b=b^{\prime} / \sigma$ on the generated wave field, let us consider the variation of the system eigenvalues $\omega_{n}$ with $b$. Recall that the $\omega_{n}$ are the positive solutions of the eigenvalue condition Eq. (63), with

$\omega_{n} \in(0, k \sqrt{b}), \quad n=0, \ldots, N(k, b)$.

Figure 11 shows the behaviour of the first four eigenfrequencies $\omega_{n}, n=0, \ldots, 3$, versus $b$ for the fixed parameter $k=1$; the bold line represents the upper limit $k \sqrt{b}$. When $b$ is very small, i.e. the domain of Eq. (85) is very narrow, only the first eigenvalue $\omega_{0}$ exists, corresponding to the first trapped mode. All eigenfrequencies larger than $\omega_{0}$ would correspond to progressive waves, which cannot be excited by a transient local perturbation (see Sect. 3.2) and must be excluded. Increasing $b, \omega_{0}$ increases and quickly reaches a limiting value, corresponding numerically to the first eigenvalue of the indefinite plane beach $\omega_{0}(k=1)=1$ (see Eq. 8). For larger values of $b$, the upper limit of the domain Eq. (85) increases as $\sqrt{b}$, thus, leaving room for higherorder eigenvalues to appear. As a consequence, the sequence of eigenvalues $\omega_{0}, \ldots, \omega_{N}, N=1,2, \ldots$ forms progressively, all the $\omega_{n}$ quickly converging to their relevant indefinite plane-beach values. For very large $b$ all the eigenvalues of the semi-plane beach $\omega_{n}$ eventually equate those of the indefinite plane beach and $N(k, b)$ grows to infinity, i.e. $\omega_{n} \rightarrow$ $\sqrt{k(2 n+1)}$ and $N(k, b) \rightarrow \infty$. Now, in the limit $b \rightarrow \infty$ the parameter $\alpha_{n}$ becomes $\alpha_{n}=1 / 2-\omega_{n}^{2} / 2 k \rightarrow-n$, and the relevant Kummer function $M\left(\xi, \alpha_{n}\right)$ Eq. (65) of the first kind and order $n$ transforms into the Laguerre polynomial $L_{n}(\xi)$, i.e. $M\left(\xi, \alpha_{n}\right) \rightarrow M(\xi,-n) \equiv L_{n}(\xi)$ (see Abramowitz and Stegun, 1972). As a consequence, for $b \rightarrow \infty$, the eigenfunctions Eq. (64) are given by $X_{n}(\xi) \rightarrow M(\xi,-n)=$ $L_{n}(\xi), \quad \xi \in(0, \infty)$, while the distinction between the near field and the far field becomes meaningless, since the geometry is that of an indefinite plane beach. Finally, note that the square norm of the eigenfunctions Eq. (A8) becomes

$$
\chi_{n}^{2}(k) \rightarrow \int_{0}^{\infty} e^{-\xi} L_{n}^{2}(\xi) d \xi=1
$$

as $b \rightarrow \infty$, so that Eq. (80) can be rewritten as

$$
\mathcal{M}^{n}[u] \rightarrow \frac{2 k}{\omega_{n}} \int_{0}^{\infty} e^{-k \rho} L_{n}(2 k \rho) u(\rho, k, t) d \rho \equiv \mathcal{L}^{n}[u],
$$

where $\mathcal{L}^{n}$ is the Laguerre integral transform Eq. (18). As a consequence, the asymptotic forms of the oscillatory and the evanescent components of the free-surface elevation, Eqs. (81) and (84), are, respectively,

$$
\begin{aligned}
& \zeta^{o}(x, y, t) \rightarrow \frac{2}{\pi} \sum_{n=0}^{\infty} \int_{0}^{\infty} e^{-k x} L_{n}(2 k x) \\
& \quad \times\left[A_{n} \cos \omega_{n} t+B_{n} \sin \omega_{n} t\right] \cos k y d k
\end{aligned}
$$

and

$$
\begin{aligned}
& \zeta^{e}(x, y, t) \rightarrow \frac{2}{\pi} \sum_{n=0}^{\infty} \int_{0}^{\infty} e^{-k x} L_{n}(2 k x) \\
& \times \omega_{n} \hat{s} \mathcal{L}^{n}\left[e^{-(\rho-t)^{2}}\right] \cos k y d k,
\end{aligned}
$$


as $b \rightarrow \infty$, where the $A_{n}$ s and $B_{n}$ s are still given by Eqs. (82) and (83), respectively, replacing $\mathcal{M}^{n}$ with $\mathcal{L}^{n}$. Now note that the asymptotic forms of the free-surface components $\zeta^{o}$ Eqs. (86) and $\zeta^{e}(87)$ coincide with the analogous expressions of the indefinite plane beach for the Gaussian slide, Eqs. (19) and (22) respectively, provided $x_{0}=0$ and $u_{0}=1$. Therefore, in the limit $b \rightarrow \infty$, the semi-plane beach model perfectly agrees with the indefinite plane beach model. Let us now investigate the influence of $b=b^{\prime} / \sigma$ on the generated wave field by analysing the tsunami propagating along a semi-plane beach. Let us first consider small $b=O(1)$ and compare the results to those obtained for the indefinite plane beach. If $b=O(1)$, then $b^{\prime} \simeq \sigma$, i.e. the characteristic length of the landslide is comparable to the horizontal length of the incline. As an example, this situation can occur for unstable rock cliffs falling into the ocean. To analyse the wave field propagating in such a geometry, let us consider the same Gaussian landslide of Sect. 2, with parameters $\sigma=0.37 \mathrm{~m}, c=\sigma / \lambda=2, x_{0}=0, u_{0}=1$, sliding along an incline of slope $s=1 / 3$ and take $b^{\prime}=\sigma$, i.e. $b=b^{\prime} / \sigma=1$ as a limit case. The solid lines of Fig. 12a, b show the time series of the free-surface elevation $\zeta^{\prime}\left(x^{\prime}, y^{\prime}, t^{\prime}\right)$ in physical variables, at points, respectively, $\left(x^{\prime}, y^{\prime}\right)=(0.4,0) \mathrm{m}$ offshore, and $\left(x^{\prime}, y^{\prime}\right)=(0,0.8) \mathrm{m}$ on the shoreline. In the same figure, the time series of the free-surface elevation obtained at the same points, but for an indefinite plane beach, are reported with dashed lines for comparison. First, note that the maximum runup and drawdown of the generated wave field are larger for the indefinite plane beach than the semiplane beach, i.e. the wave field generated in the semi-plane beach is less energetic. This happens since for small $b$ only the very first eigenmodes are excited and concur to generate the propagating wave field. Physically, having required the forcing function $f$ to annihilate in the far field, determines the landslide mass to disappear suddenly as it reaches the boundary $x=b$. The reduced time of interaction between landslide and water for small $b$ is therefore responsible for a strong decrease in the amount of the total energy yielded by the landslide to the water, especially in the offshore direction (see Fig. 12a). This determines the reduction in amplitude of the generated waves in the semi-plane beach. However this does not imply loss of physical meaning for this case: the landslide accumulates and stops at the toe of the slope. Now let us consider the case $b \gg 1$, or in physical variables $b^{\prime} \gg \sigma$, i.e. the characteristic length of the slide is small if compared to the horizontal length of the incline. Physically, this situation represents a landslide involving only a small part of the beach. As an example, here we consider the same slide of the previous subsection, but increase the non-dimensional horizontal length of the incline to the value $b=10$. Figs. $13 \mathrm{a}, \mathrm{b}$ represent the time series of the free-surface elevation in non-dimensional variables both for the semi-plane beach (solid line) and the indefinite beach (dashed line) at points $\left(x^{\prime}, y^{\prime}\right)=(0.4,0) \mathrm{m}$ and $\left(x^{\prime}, y^{\prime}\right)=(0,0.8) \mathrm{m}$, respectively. Note that for $b=10$ the semi-plane beach and the indefinite beach models agree almost completely, i.e. the influence of the flat bottom on the generated wave field is negligible. In such cases, application of the plane beach model is preferable, due to its easiness in calculations and to the availability of a closed form for the free-surface elevation at large times, obtained via the method of the stationary phase.

\section{Conclusions}

This work consists of two sections. In the first one, the analytical forced 2HD theory of Sammarco and Renzi (2008), on tsunamis generated by a double Gaussian landslide of unit speed and propagating along a plane beach, is extended to slides of more complex shape and dynamics. The wave field generated by an infinite double Gaussian slide of arbitrary velocity and initial position is investigated and compared to the wave field generated by a double parabolic slide of finite length, again having arbitrary velocity and initial position. For both slides, the generated wave field consists of an evanescent component travelling offshore, quickly decaying with time, and a system of transient travelling edge waves along the coastline. For a mild slope and a thin slide, the source-related evanescent terms show the same rate of decay $O\left(t^{-2}\right)$ for either slide, regardless of its shape. At large times the offshore motion decays and only transient edge waves travelling along the beach are present, their rate of decay being $O\left(t^{-1 / 2}\right)$. These waves show a clear dispersive behaviour, with longer waves travelling faster, followed by a tail of shorter waves. Larger waves are shifted towards the middle of the group, due to the difference between phase and group velocities of the transient wave trains. Dissimilarities arise in the shape of the waves generated by the two slides and propagating in the far field. The wave field originating from the Gaussian slide is smooth and regular, while the perturbation generated by the finite-length double parabolic slide is characterised by the occurrence of spiky, double-crested waves. These double crested waves are an effect of the secondary inertial rebound occurring at the origin soon after the tail of the slide enters water, which cannot be rendered by employing the more ancillary double Gaussian shape. Hence, the finiteness of the landslide is an important feature that cannot be neglected in analytical models aiming to reproduce accurately the shape of the generated waves.

In the second part, an analytical forced 2HD model has been developed to analyse the distinguishing features of landslide tsunamis on a semi-plane beach. The solution obtained via the method of separation of variables only allows the existence of trapped wave modes propagating along the coast. For very large values of the horizontal length of the slope, the free-surface elevation matches the solution obtained for an infinite sloping beach, where all the eigenmodes are trapped. For a finite horizontal length of the slope, a smaller number of eigenmodes are excited. The free-surface 
elevation is the sum of an evanescent component, quickly decaying with time, and an oscillatory component travelling along the shoreline. Despite the presence of the constantdepth region would suggest the occurrence of an offshoretravelling wave train, no wave is radiated offshore. As a consequence, energy is trapped along the coastline, but a smaller number of spectral components are excited with respect to an infinitely long beach. In this sense, the presence of the continental platform may be beneficial in mitigating the severity of the tsunami.

\section{Appendix A}

\section{Orthogonality of the semi-plane beach natural modes}

It is a well-known result that the spatial eigenfunctions of the plane beach problem, namely the Laguerre polynomials $L_{n}$, are orthogonal with respect to the weighting function $e^{-\xi}$ (see Abramowitz and Stegun, 1972), i.e. $\int_{0}^{\infty} e^{-\xi} L_{n}(\xi) L_{m}(\xi) d \xi=\delta_{n m}$. In this subsection we wonder if a similar property still holds for the spatial eigenfunctions $X_{n}$ Eq. (64) of the semi-plane beach problem. To perform this investigation, recall that the $X_{n} \mathrm{~s}$ are the independent solutions of Eq. (48); in other words, they satisfy

$h X_{n, \xi \xi}+\left(h_{\xi}-h\right) X_{n, \xi}+\left(\frac{\omega_{n}^{2}}{4 k^{2}}-\frac{h_{\xi}}{2}\right) X_{n}=0$,

with $\omega_{n}$ the system eigenvalues solving Eq. (63). Now, let us reduce Eq. (A1) to its corresponding Sturm-Liouville form

$\left[h(\xi) e^{-\xi} X_{n, \xi}\right]_{\xi}-\left(\frac{h_{\xi}(\xi)}{2}-\frac{\omega_{n}^{2}}{4 k^{2}}\right) e^{-\xi} X_{n}=0$.

Then consider two independent solutions of Eq. (A2), $X_{p}$ and $X_{q}$, of eigenvalues $\omega_{p}$ and $\omega_{q}$ respectively, with $p, q \in \mathbb{N}$ and $p \neq q$. If we first write Eq. (A2) for $X_{p}$ and for $X_{q}$ separately, multiply the first equation by $X_{q}$, the second by $X_{p}$ and then subtract the two of them, we get

$\left[h e^{-\xi} X_{p, \xi}\right]_{\xi} X_{q}-\left[h e^{-\xi} X_{q, \xi}\right]_{\xi} X_{p}=\left(\frac{\omega_{p}^{2}-\omega_{q}^{2}}{4 k^{2}}\right) e^{-\xi} X_{p} X_{q}$.

The latter expression can be further simplified by using the differential form

$$
\begin{array}{r}
\frac{d}{d \xi}\left[h e^{-\xi}\left(X_{q} X_{p, \xi}-X_{p} X_{q, \xi}\right)\right] \\
=\left(\frac{\omega_{p}^{2}-\omega_{q}^{2}}{4 k^{2}}\right) e^{-\xi} X_{p} X_{q} .
\end{array}
$$

Integrating Eq. (A3) in all the fluid domain, $\xi \in(0, \infty)$, yields

$$
\begin{array}{r}
\frac{\omega_{p}^{2}-\omega_{q}^{2}}{4 k^{2}} \int_{0}^{\infty} e^{-\xi} X_{p} X_{q} d \xi \\
=\int_{0}^{\infty} \frac{d}{d \xi}\left[h e^{-\xi}\left(X_{q} X_{p, \xi}-X_{p} X_{q, \xi}\right)\right] .
\end{array}
$$

To solve the latter integral, express the bottom depth $h$ Eq. (42) at the right-hand side in terms of the transformed variable $\xi$ Eq. (45): $h(\xi)=\xi / 2 k$ if $\xi<2 k b$ and $h(\xi)=b$ if $\xi \geq 2 \mathrm{~kb}$. Note that $h$ is a continuous function of $\xi$; also, continuity of the free-surface elevation and of the fluxes in Eq. (62) determines $X(\xi)$ and $X_{\xi}(\xi)$ to be continuous over the entire domain $\xi \in(0, \infty)$. As a result, the integrands at both sides of Eq. (A4) are continuous functions of $\xi$. Solving the integral at the right-hand side of Eq. (A4) gives then

$\frac{\omega_{p}^{2}-\omega_{q}^{2}}{4 k^{2}} \int_{0}^{\infty} e^{-\xi} X_{p} X_{q} d \xi=0$,

since $h(0)=0$ and by virtue of Eq. (64)

$\lim _{\xi \rightarrow+\infty} h(\xi) e^{-\xi}\left(X_{q} X_{p, \xi}-X_{p} X_{q, \xi}\right)=0$.

As a consequence, Eq. (A5) simply becomes

$\int_{0}^{\infty} e^{-\xi} X_{n}(\xi) X_{m}(\xi) d \xi=0 \quad$ if $n \neq m$.

Let us now define the square norm of the eigensolutions $X_{n}$ Eq. (64) as

$$
\begin{aligned}
\chi_{n}^{2}(k)=\int_{0}^{\infty} e^{-\xi} X_{n}^{2}(\xi) d \xi= & \int_{0}^{2 k b} e^{-\xi} M^{2}\left(\xi, \alpha_{n}\right) d \xi \\
& +\frac{e^{-2 k b} M^{2}\left(2 k b, \alpha_{n}\right)}{\beta_{n}} ;
\end{aligned}
$$

hence, coupling Eqs. (A7) and (A8) yields the orthogonality relation satisfied by the spatial eigenfunctions $X_{n}$ of the governing Eq. (48)

$\int_{0}^{\infty} e^{-\xi} X_{n}(\xi) X_{m}(\xi) d \xi=\chi_{n}^{2} \delta_{n m}$.

\section{Appendix B}

\section{Computational aspects}

In Sect. 2 the solution of the plane beach problem was found in terms of the oscillatory and evanescent components of the generated perturbation, given respectively by Eqs. (19) and (22) for the double Gaussian landslide and by Eqs. (23) and (27) for the double parabolic landslide. Mathematically, these expressions involve calculation of an infinite series of two nested integrals over infinite domains and posed several computational challenges. Numerical evaluation of the integrals was performed via a modified Gauss-Laguerre integration method (Renzi, 2010, see for details). The latter is a quadrature method whose nodal abscissas are the zeros of the Laguerre polynomials and proves to be very efficient for the calculation of integrals with an exponentially convergent integrand. A number of 60 Gauss points is sufficient to ensure convergence of the integrals treated in this work with largest relative error $O\left(10^{-3}\right)$. The rate of convergence of 
the outer summations in the full solutions $\zeta_{g}$ and $\zeta_{p}$ depends on the parameters of the system. For the geometry considered here, about 10 modes were necessary to evaluate the free-surface elevation at different times and points, with an average relative error of about $10 \%$. This is enough precision to provide an estimate of the general behaviour of the fluid. Extension of the summation up to 20 terms allows a significant refinement of the results, with an average relative error now less than $3 \%$. Convergence of the outer series is much faster when considering the stationary phase approximation Eq. (34). Here, about 5 terms are already sufficient to obtain an average relative error of about $1 \%$. Usually, convergence was faster for the double Gaussian slide than for the double parabolic one. This is likely due to the singularity introduced by the Heaviside step function at the borders of the double parabolic slide (see Eq. 12). Similar figures also apply to the solution of the semi-plane beach problem in Sect. 3.

Acknowledgements. This work was funded by the Italian Ministry of Research (MIUR) under the research project "Development and validation of hydraulic and geologic tools for supporting a Tsunami Early Warning System. Implementation to the Stromboli (Eolie) landslide case" (PRIN-2007). Experimental data provided by M. Di Risio have been very useful for experimental comparison. Fruitful discussions with Giorgio Bellotti are kindly acknowledged. The work of the skillful students Mathieu Lecouvez, Camille Grosso and Theo Le Bars of ENPC (Paris) was very helpful for the analytical calculations.

Edited by: S. Tinti

Reviewed by: two anonymous referees

\section{References}

Abramowitz, M. and Stegun, I. : Handbook of mathematical functions, Dover Publications, Mineola, USA, 1972.

Bellotti G., Cecioni, C. and De Girolamo, P.: Simulation of smallamplitude frequency-dispersive transient waves by means of the mild-slope equation, Coast. Eng., 55, 447-458, 2008.

Cheng, H.: Advanced Analytic Methods in Applied Mathematics, Science and Engineering, LuBan Press, Boston, USA, 2007.
Di Risio, M., Bellotti, G., Panizzo, A., and De Girolamo, P.: Threedimensional experiments on landslide generated waves at a sloping coast, Coast. Eng., 56, 5-6, 659-671, 2009.

Gonzalez, F. I., Satake, K., Boss, E. F., and Mofjeld, H. O.: Edge Wave and Non-Trapped Modes of the 25 April 1992 Cape Mendocino Tsunami, Pure Appl. Geophys., 144, 409-426, 1995.

Li, R., Liu, S., Guan, Q., and Peng, Y.: Post-disaster Assessment of Northeastern Coastal Region for the 2011 Sendai Earthquake and Tsunami, ISWREP 2011 - Proceedings of 2011 International Symposium on Water Resource and Environmental Protection, 3, 2429-2432, 2011.

Liu, P. L.-F., Lynett, P., and Synolakis, C.-E.: Analytical solutions for forced long waves on a sloping beach, J. Fluid Mech., 478, 101-109, 2003.

Liu, P. L.-F., Wu, T. R., Raichlen, F., Synolakis, C.-E., and Borrero, J. C.: Runup and rundown generated by three-dimensional sliding masses, J. Fluid Mech., 536, 107-144, 2005.

Longuet-Higgins, M. S.: On the trapping of wave energy round islands, J. Fluid Mech., 29, 781-821, 1967.

Lynett, P. and Liu, P. L.-F.: A numerical study of the runup generated by three-dimensional landslides, J. Geophys. Res., 110, C03006, doi:10.1029/2004JC002443, 2005.

Mei, C. C.: Mathematical analysis in engineering, Cambridge University Press, New York, USA, 1997.

Mei, C. C., Stiassnie, M., and Yue, D.: Theory and application of ocean surface waves, World Scientific, New York, USA, 2005.

Meyer, R. E.: Resonance of unbounded water bodies, Lect. Appl. Math., AMS, USA, 190-227, 1971.

Renzi, E.: Landslide Tsunamis, Ph.D. Thesis, Università degli Studi di Roma Tor Vergata, Italy, 2010.

Sammarco, P. and Renzi, E.: Landslide tsunamis propagating along a plane beach, J. Fluid Mech., 598, 107-119, 2008.

Sarri, A., Guillas, S., and Dias, F.: Statistical emulation of a tsunami model for sensitivity analysis and uncertainty quantification, accepted, Nat. Hazards Earth Syst. Sci., 2012.

Watts, P., Grilli, S. T., Kirby, J. T., Fryer, G. J., and Tappin, D. R.: Landslide tsunami case studies using a Boussinesq model and a fully nonlinear tsunami generation model, Nat. Hazards Earth Syst. Sci., 3, 391-402, doi:10.5194/nhess-3-391-2003, 2003. 